\title{
PCCP
}

\section{A DFT study of 2-aminopurine-containing dinucleotides: prediction of stacked conformations with B-DNA structure}

Darren A. Smith $+^{+a}$ Leo F. Holroyd $+^{\mathrm{b}}$ Tanja van Mourik ${ }^{\mathrm{b}}$ and Anita C. Jones*a

\section{Supplementary Information}

Table S1 Base-step parameters obtained by analysis of structural data reported for $\mathrm{d}(\mathrm{GpN})$ and $\mathrm{d}(\mathrm{ApN})$ dinucleotides in the Supplementary Information of Barone et al. ${ }^{1}$ Values for idealised B-DNA and mean values from B-DNA crystal structures are presented for comparison.

\begin{tabular}{|c|c|c|c|c|c|c|}
\hline & Shift /Å & Slide /Å & Rise / $\AA$ & Tilt $/{ }^{\circ}$ & Roll / ${ }^{\circ}$ & Twist / \\
\hline$\overline{\text { B-DNA Ideal }{ }^{a}}$ & 0.00 & 0.00 & 3.34 & 0.00 & 0.00 & 36.00 \\
\hline B-DNA Mean ${ }^{b}$ & -0.02 & 0.23 & 3.32 & -0.10 & 0.60 & 36.00 \\
\hline (Std. Dev.) & $(0.45)$ & $(0.81)$ & $(0.19)$ & $(2.50)$ & $(5.20)$ & $(6.80)$ \\
\hline $\mathrm{d}(\mathrm{GpA})$ & 1.13 & -0.15 & 3.17 & 0.75 & 1.02 & 53.77 \\
\hline$d(\mathrm{ApA})$ & 3.55 & 1.35 & 3.02 & 7.49 & 10.70 & 53.98 \\
\hline $\mathrm{d}(\mathrm{GpG})$ & 1.61 & 0.57 & 3.12 & 10.91 & -6.72 & 58.84 \\
\hline $\mathrm{d}(\mathrm{ApG})$ & 3.32 & 2.59 & 3.10 & 18.64 & 8.06 & 62.64 \\
\hline $\mathrm{d}(\mathrm{GpC})$ & 0.52 & -1.36 & 3.72 & -9.01 & 0.99 & 46.12 \\
\hline $\mathrm{d}(\mathrm{ApC})$ & 0.47 & -0.29 & 3.46 & -3.35 & 0.48 & 37.66 \\
\hline $\mathrm{d}(\mathrm{GpT})$ & -1.13 & -0.35 & 3.70 & -4.19 & -16.01 & 22.42 \\
\hline $\mathrm{d}(\mathrm{ApT})$ & 0.53 & -0.04 & 3.22 & 2.15 & -5.57 & 35.18 \\
\hline
\end{tabular}

Table S2 Base-step parameters for computed structures of twisted $\mathrm{d}(2 \mathrm{pN})$ dinucleotides and the corresponding $2 \mid \mathrm{N}$ dimers.

\begin{tabular}{|c|c|c|c|c|c|c|}
\hline & Shift /Å & Slide /Å & Rise / & Tilt $/{ }^{\circ}$ & Roll / ${ }^{\circ}$ & Twist $/{ }^{\circ}$ \\
\hline$d(2 p A)$ & 1.53 & -0.13 & 3.14 & 0.46 & -0.32 & 60.60 \\
\hline $2 \mid \mathrm{A}$ & 1.55 & -0.21 & 3.11 & -0.12 & 1.66 & 58.45 \\
\hline $\mathrm{d}(2 \mathrm{pG})$ & 1.46 & -0.18 & 3.07 & 4.74 & -4.05 & 59.12 \\
\hline $2 \mid \mathrm{G}$ & 1.57 & -0.23 & 3.05 & 3.86 & -3.76 & 59.56 \\
\hline $\mathrm{d}(2 \mathrm{pC})$ & 0.49 & -1.54 & 3.68 & -8.75 & -1.19 & 45.61 \\
\hline $2 \mid \mathrm{C}$ & -0.89 & -2.30 & 3.12 & 1.87 & -2.04 & 17.47 \\
\hline $\mathrm{d}(2 \mathrm{pT})$ & -0.14 & -1.55 & 3.43 & -2.13 & -5.92 & 38.81 \\
\hline $2 \mid \mathrm{T}$ & -0.85 & -2.46 & 3.16 & 1.19 & -3.30 & 15.54 \\
\hline
\end{tabular}

a. EaStCHEM School of Chemistry, The University of Edinburgh, David Brewster Road, Edinburgh, EH9 3FJ, UK. E-mail: a.c.jones@ed.ac.uk

b. EaStCHEM School of Chemistry, University of St Andrews, North Haugh, St Andrews, Fife, KY16 9ST, UK.

† The authors wish it to be known that, in their opinion, the first 2 authors should be regarded as joint First Authors 
Table S3 Base-step parameters for computed structures of twisted $\mathrm{d}(\mathrm{ApN})$ dinucleotides and the corresponding $\mathrm{A} \mid \mathrm{N}$ dimers.

\begin{tabular}{|c|c|c|c|c|c|c|}
\hline & Shift /Å & Slide /Å & Rise / & Tilt $/{ }^{\circ}$ & Roll / ${ }^{\circ}$ & Twist $/{ }^{\circ}$ \\
\hline $\mathrm{d}(2 \mathrm{pA})$ & 1.21 & -0.05 & 3.24 & -2.49 & 0.43 & 51.53 \\
\hline $\mathrm{A} \mid \mathrm{A}$ & 1.43 & -0.22 & 3.12 & -0.44 & 3.80 & 55.91 \\
\hline $\mathrm{d}(\mathrm{ApG})$ & 1.45 & -0.11 & 3.19 & 0.53 & -3.59 & 50.13 \\
\hline $\mathrm{A} \mid \mathrm{G}$ & 1.44 & -0.33 & 3.08 & 2.40 & 1.53 & 42.89 \\
\hline $\mathrm{d}(\mathrm{ApC})$ & -0.13 & -1.17 & 3.67 & -9.10 & 6.62 & 40.42 \\
\hline $\mathrm{A} \mid \mathrm{C}$ & 2.44 & -1.86 & 3.06 & 0.85 & 3.06 & 75.02 \\
\hline $\mathrm{d}(\mathrm{ApT})$ & -0.70 & -1.20 & 3.22 & 0.79 & -3.17 & 33.81 \\
\hline $\mathrm{A} \mid \mathrm{T}$ & 2.16 & -2.03 & 2.92 & 5.56 & -2.27 & 69.20 \\
\hline
\end{tabular}

Table S4 Sugar pucker parameters for computed twisted structures of $d(2 \mathrm{pN})$ and $d(\mathrm{ApN})$ dinucleotides in comparison with those of ideal B-DNA. P is the phase angle of pseudorotation. ${ }^{a}$

\begin{tabular}{|c|c|c|c|c|}
\hline & \multicolumn{2}{|c|}{ 5'-Base } & \multicolumn{2}{|c|}{ 3'-Base } \\
\hline & $\mathrm{P} /{ }^{\circ}$ & Pucker & $\mathrm{P} /{ }^{\circ}$ & Pucker \\
\hline B-DNA Ideal ${ }^{b}$ & $144-180$ & C2'-endo & $144-180$ & C2'-endo \\
\hline$d(2 p A)$ & 170.9 & C2'-endo & 2.6 & C3'-endo \\
\hline $\mathrm{d}(\mathrm{ApA})$ & 181.6 & C3'-exo & 12.8 & C3'-endo \\
\hline$d(2 p G)$ & 169.9 & C2'-endo & 2.2 & C3'-endo \\
\hline $\mathrm{d}(\mathrm{ApG})$ & 183.3 & C3'-exo & 15.7 & C3'-endo \\
\hline$d(2 p C)$ & 137.5 & C1'-exo & 22.0 & C3'-endo \\
\hline $\mathrm{d}(\mathrm{ApC})$ & 136.3 & C1'-exo & 21.3 & C3'-endo \\
\hline$d(2 p T)$ & 146.0 & C2'-endo & 26.7 & C3'-endo \\
\hline$d(A p T)$ & 149.1 & C2'-endo & 25.9 & C3'-endo \\
\hline
\end{tabular}

${ }^{a}$ The range of $\mathrm{P}$ values corresponding to each conformational form is as follows: $04^{\prime}$-endo, $72-108^{\circ} ; \mathrm{C1}^{\prime}$-exo, $108-144^{\circ}$; $\mathrm{C}^{\prime}$-endo, $144-180^{\circ}$; $\mathrm{C} 3^{\prime}$-exo, $180-216^{\circ}$. ${ }^{b}$ From Altona and Sundaralingam. ${ }^{4}$

Table S5 Base-step parameters for computed unstacked structures of $\mathrm{d}(2 \mathrm{pN})$ and $\mathrm{d}(\mathrm{ApN})$ dinucleotides.

\begin{tabular}{|c|c|c|c|c|c|c|}
\hline & Shift /Å & Slide /Å & Rise / & Tilt $/^{\circ}$ & Roll / ${ }^{\circ}$ & Twist / ${ }^{\circ}$ \\
\hline $\mathrm{d}(2 \mathrm{pG})$ & -17.69 & -5.67 & 1.55 & 44.54 & -38.16 & -148.06 \\
\hline$d(A p G)$ & -17.42 & -5.86 & 2.16 & 42.17 & -41.74 & -152.49 \\
\hline $\mathrm{d}(2 \mathrm{pT})$ & -17.98 & -5.59 & 0.99 & 43.32 & -32.05 & -146.75 \\
\hline $\mathrm{d}(\mathrm{ApT})$ & -17.96 & -5.72 & 1.20 & 42.85 & -30.97 & -149.47 \\
\hline
\end{tabular}

Table $\mathbf{S 6}$ Backbone torsional angles (degrees) for computed unstacked structures of $d(2 \mathrm{pN})$ and $d(\mathrm{ApN})$ dinucleotides in comparison with mean values from B-DNA crystal structures. Standard deviations in the latter are small (<1.2) and are not shown. Torsional angles are defined in Figure 1 (main text).

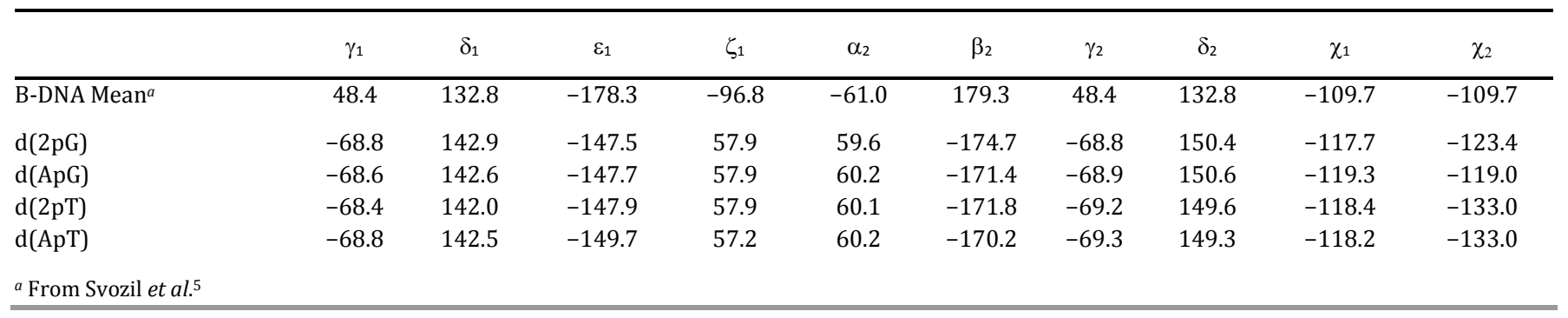


Table S7 Sugar pucker parameters for computed unstacked structures of $d(2 \mathrm{pN})$ and $d(\mathrm{ApN})$ dinucleotides in comparison with those of ideal B-DNA. P is the phase angle of pseudorotation. ${ }^{a}$

\begin{tabular}{|c|c|c|c|c|}
\hline & \multicolumn{2}{|c|}{ 5'-Base } & \multicolumn{2}{|c|}{ 3'-Base } \\
\hline & $\mathrm{P} /{ }^{\circ}$ & Pucker & $\mathrm{P} /{ }^{\circ}$ & Pucker \\
\hline B-DNA Ideal ${ }^{b}$ & $144-180$ & C2'-endo & $144-180$ & C2'-endo \\
\hline $\mathrm{d}(2 \mathrm{pG})$ & 155.1 & C2'-endo & 168.6 & C2'-endo \\
\hline$d(A p G)$ & 154.8 & C2'-endo & 169.2 & C2'-endo \\
\hline $\mathrm{d}(2 \mathrm{pT})$ & 153.8 & C2'-endo & 167.2 & C2'-endo \\
\hline$d(A p T)$ & 154.9 & C2'-endo & 166.3 & C2'-endo \\
\hline
\end{tabular}

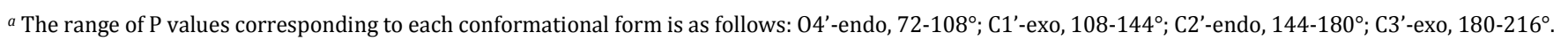
${ }^{b}$ From Altona and Sundaralingam. ${ }^{4}$

Figure S1 Comparison of the optimised structures of the twisted conformers of the dinucleotides and the optimised structures of the unbound dimers. The structure of each dinucleotide (red) is overlaid with the structure of the corresponding dimer (blue). (a) $d(2 \mathrm{pN}$ ) dinucleotides (red) and $2 \mid \mathrm{N}$ dimers (blue). (b) $d(A p N)$ dinucleotides (red) and $\mathrm{A} \mid \mathrm{N}$ dimers (blue). In each case two alternative views are shown: perpendicular to the backbone (top row) and looking along the backbone from the $5^{\prime}$ end (bottom row).

A

(a)
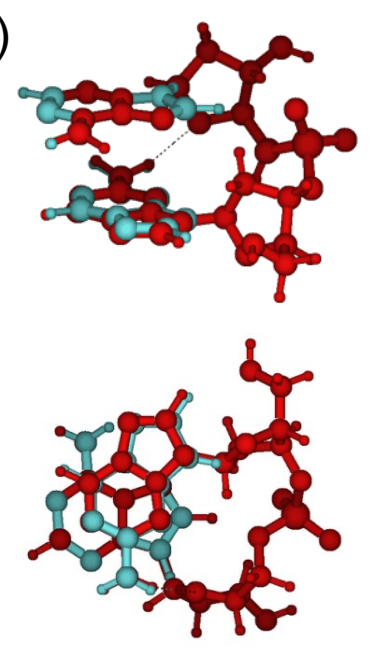

(b)
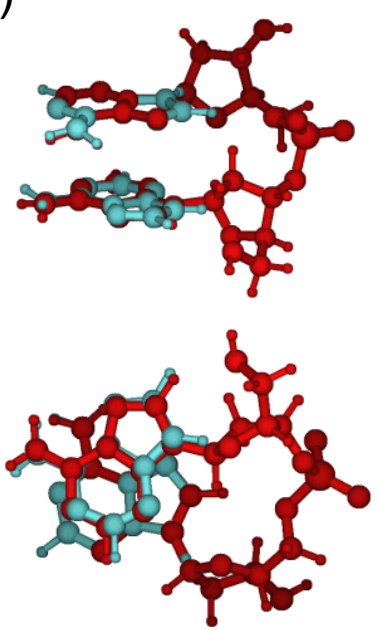

G
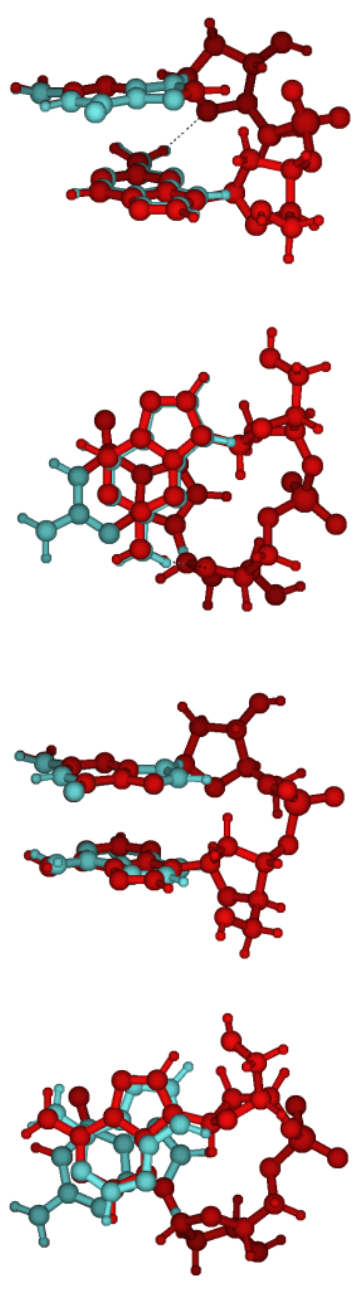

C
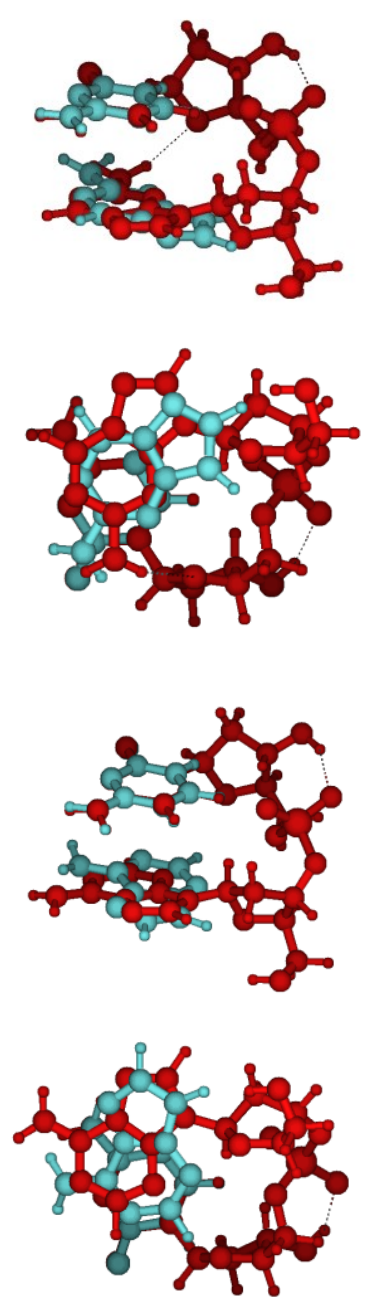

T
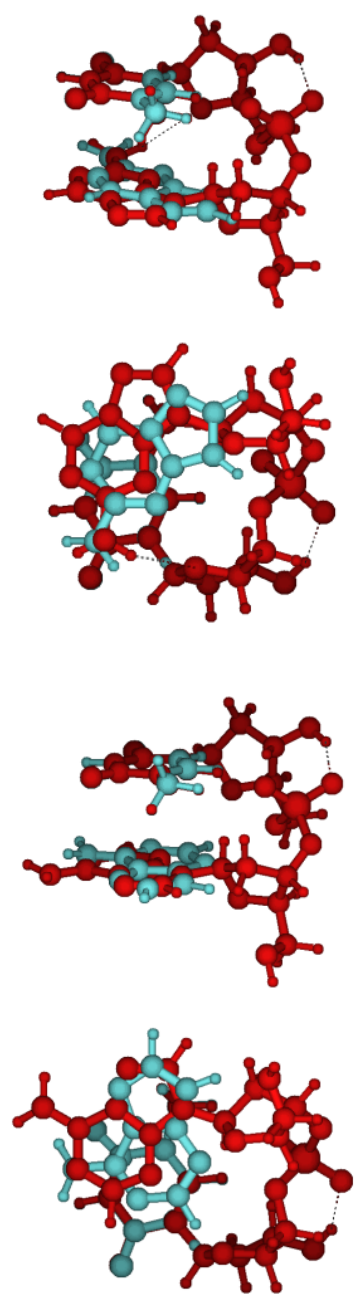


\section{Cartesian coordinates of all optimised minima}

The Cartesian coordinates are given in xyz format (first line: number of atoms; second line: title; following lines: atom symbol and Cartesian coordinates in Hartree). The title line gives information on the system (d(XpY): dinucleoside monophosphate containing bases X and Y; W|Z: dimer containing bases W and Z; d: deoxy; p: phosphate; 2: 2-aminopurine; A: adenine; C: cytosine; G: guanine; T: thymine; B-form: stacked structure in B-DNA conformation; Twisted: stacked structure in twisted conformation; Unstacked: open conformation; Dimer: dimer structure; pcm: polarizable continuum model, water).

63

$\mathrm{d}(2 \mathrm{pA})-\mathrm{B}-\mathrm{form}-\mathrm{pcm}$

\begin{tabular}{|c|c|c|}
\hline-3.02107200 & -4.22093000 & 0.10435000 \\
\hline-2.18111000 & -4.21393700 & -1.04275200 \\
\hline-2.99040700 & -2.84629800 & 38900 \\
\hline-1.70531500 & -2.58707900 & 1.3248070 \\
\hline 36200 & -1.70051600 & -0.24 \\
\hline-3.95730900 & -0.68586400 & 0.4681520 \\
\hline 825600 & -1.25503200 & -0.62 \\
\hline-1.02477100 & -1.54680900 & 0.6371720 \\
\hline 051600 & -0.97796800 & 1.8086520 \\
\hline 2.87120200 & -0.27385300 & 2.193831 \\
\hline 1.60324500 & -0.48297900 & 1.81 \\
\hline 1.47807600 & -1.49239400 & 0.966768 \\
\hline 2.52201500 & -2.29873600 & 0.47 \\
\hline 3.78983000 & -1.98726000 & 0.956614 \\
\hline 2.02601800 & -3.25887000 & -0.390324 \\
\hline 0.74021200 & -3.03119500 & -0.41895 \\
\hline 0 & -1.97242800 & \\
\hline-4.47624200 & 0.65465300 & -0.336778 \\
\hline-2.77 & 2.10152500 & 1.0 \\
\hline-3.14859400 & 1.62189300 & -0.2 \\
\hline-1.4 & 2.84083800 & 0.9 \\
\hline-0.41631800 & 1.90479500 & 0.63 \\
\hline-1.41 & 3.91646900 & -0.1 \\
\hline-0.88 & 5.14712300 & 0.2 \\
\hline-0 . & 3.33977100 & $-1 \cdot 1$ \\
\hline 0.49 & 2.54102700 & -0.2 \\
\hline 5.25 & 0.68297700 & -0.7 \\
\hline 4.77643500 & 1.71883500 & -0.07 \\
\hline 3.52 & 2.14 & 0.0 \\
\hline 2.68 & 1.39764700 & -0.7 \\
\hline 3.03 & 0.29 & $-1 \cdot 4$ \\
\hline 4.39 & -0.06137 & -1.4 \\
\hline 4. & -1.11 & -2.1 \\
\hline 1.92 & -0.22936 & -2.11 \\
\hline 0.9 & 0.54 & $-1 \cdot 7$ \\
\hline 1.32 & 1.544330 & -0.869 \\
\hline-5 . & 1.24 & 0.51 \\
\hline-4.65 & 0.33 & -1.789 \\
\hline 0 & -3.60 & -0.971 \\
\hline-0.93 & -0.66662700 & 1.281 \\
\hline-1 . & -1.85 & -1.463 \\
\hline-1.80 & -0.19581200 & -0.882 \\
\hline-3 . & -2.0197 & -1.107 \\
\hline-3.73 & -2.8195730 & 1.545 \\
\hline-2.676 & -4.9622210 & 0.835 \\
\hline-4 & -4.45497 & -0.174 \\
\hline-2 . & -5.08996 & $-1 \cdot 4$ \\
\hline-2 . & 1.26369 & 1.733 \\
\hline
\end{tabular}




\begin{tabular}{|c|c|c|}
\hline-3 & 2 & 1 \\
\hline & 3.32522000 & 1.887201 \\
\hline-2.39941700 & 4.07147100 & -0.6462510 \\
\hline-0.90476200 & 2.66105100 & -1.8771 \\
\hline 0.12586800 & 4.11608500 & -1.7337840 \\
\hline 1.17714600 & 3.19636300 & 0.3143660 \\
\hline-1.52076300 & 5.55122700 & 0.89 \\
\hline 881300 & 2.27947500 & 0.45 \\
\hline 4.18225100 & -1.79891100 & -2.53 \\
\hline 5.786 & -1.44685 & -2.0 \\
\hline-0.09062500 & 0.43470000 & -2.0 \\
\hline 3. & 0.7273 & 3. \\
\hline 4.03 & 1.08 & 3.1 \\
\hline 2 . & 1.45 & 3.1 \\
\hline & $-2 \quad 54$ & \\
\hline$-\mathrm{B}$ & & \\
\hline-2 . & -4.32 & 0.2 \\
\hline-1 . & -4.27 & -0.9 \\
\hline-2 . & -2.93 & 0.8 \\
\hline$-1 \cdot 22$ & -2.58 & 1.4 \\
\hline-2 & -1.82 & -0.1 \\
\hline & -0.86 & \\
\hline-1.50 & $-1.2 \varepsilon$ & -0.5 \\
\hline-0 & -1.4 & 0.6 \\
\hline 4.32 & -0.57 & 1.7 \\
\hline 3. & 0 . & \\
\hline 1.94 & -0.22 & 1.8 \\
\hline 1 . & -1.24 & \\
\hline 2.94 & -1.98 & 0.4 \\
\hline 4. & -1.59 & 0 . \\
\hline 2.48 & -2.98 & -0 \\
\hline 1 . & -2.84 & -0 \\
\hline 0.73 & -1.81 & 0.4 \\
\hline-4 . & 0.39 & -0 \\
\hline-2.63 & 2.00 & 0 . \\
\hline-3.08 & 1.46 & -0.2 \\
\hline$-1 \cdot 30$ & 2.70 & 0.7 \\
\hline-0 . & 1.74 & \\
\hline-1.26 & 3.80 & -0.3 \\
\hline-1.27 & 5.10 & \\
\hline 0 . & 3.56 & -1.0 \\
\hline 0 . & 2.50 & -0 \\
\hline 1.65 & 1.61 & -0.8 \\
\hline 3. & 1.71 & -0 \\
\hline 3. & 2.58 & 0 . \\
\hline $3 . \varepsilon$ & 0.82 & $-1 \cdot 2$ \\
\hline 3.36 & -0.09486700 & -2.0 \\
\hline 1.96593000 & $-0.1 \varepsilon$ & $-2 \cdot 3$ \\
\hline 9520 & 0.70109700 & -1.7 \\
\hline-5 & 0 . & 0.6 \\
\hline-4 & -0.00676000 & -1.657 \\
\hline 0.4 & -3.47622 & -0 \\
\hline 207460 & -0.60404600 & 1.333 \\
\hline-1 . & $-1 \cdot 8$ & -1 \\
\hline-1 . & -0.227567 & -0 \\
\hline 39 & - & \\
\hline
\end{tabular}




\begin{tabular}{|c|c|c|c|}
\hline & -3.23220700 & -2.95261300 & 1.67819800 \\
\hline & -2.04231100 & -5.02537400 & 0.96975000 \\
\hline & -3.47778900 & -4.63031100 & -0.01348800 \\
\hline & -1.61696500 & -5.15235300 & -1.33 \\
\hline & -2.51243300 & 1.20846600 & 1.68028500 \\
\hline & -3.36395200 & 2.72949000 & 1.32525900 \\
\hline & -1.00697300 & 3.16240500 & 168537500 \\
\hline & -2.10912000 & 3.70245700 & -1.04 \\
\hline & -0.08377400 & 3.16195900 & -2.06018400 \\
\hline & 0.65614200 & 4.48438000 & -1.13240300 \\
\hline & 1.32241600 & 2.96187300 & 0.63843800 \\
\hline & -2.16563600 & 5.31486200 & 0.53760300 \\
\hline & 0.0 & 2422600 & $-1.88<-2$ \\
\hline & 4.22805200 & -0.95281600 & -2.6 \\
\hline & 84800 & 913300 & -2 . \\
\hline & 3.88883800 & -1.77683700 & -3.12125200 \\
\hline & 1.57203000 & -0.92586500 & -3.06000800 \\
\hline & 3.3893 & 500 & \\
\hline & 4.3 & 0 & 3. \\
\hline & 2.63466900 & 1.74953700 & 3.08 \\
\hline & 5.10964500 & -2.08849500 & 0.55795100 \\
\hline & (1) & & \\
\hline & -3.39890400 & -4.06535300 & 0.16079400 \\
\hline & -2.6 & 32900 & $-1 \cdot 0$ \\
\hline & -3.25680100 & -2.70342300 & 0.81199500 \\
\hline & -1.94159100 & -2.53623200 & 1.35858700 \\
\hline & -3.46641600 & -1.53321800 & -0.15495000 \\
\hline & -4 & -0.49074100 & 0.57 \\
\hline & -2.04306800 & -1.16092000 & -0.5 \\
\hline & -1.21258800 & -1.53275500 & 0.66656000 \\
\hline & 3.85914300 & -1.34881600 & 1.61192300 \\
\hline & 2.84716600 & -0.56589900 & 2.05364600 \\
\hline & 1.549819 & -0.67427100 & 1.73972 \\
\hline & 1.3 & 751400 & \\
\hline & 2.25794700 & -2.55634900 & 58200 \\
\hline & 3.56779700 & -2.34330300 & 0.77379800 \\
\hline & 1.64957100 & -3.47481800 & -0.47848900 \\
\hline & 0.38724600 & -3.14217200 & -0.45486600 \\
\hline & 0.1 & -2.05226000 & 00 \\
\hline & -4.61529 & 0 . & -0 . \\
\hline & -2.793011 & 2.23790700 & 396900 \\
\hline & -3.24877200 & 1.77604500 & -0.20093300 \\
\hline & -1.4392960 & 2.88549400 & 0.89117400 \\
\hline & -0.471082 & 1.87691900 & 0.57885900 \\
\hline & -1.36878 & 3818400 & -0.24842800 \\
\hline & -0.733511 & 5.13561100 & 0.18199100 \\
\hline & -0.44928600 & 3.27605900 & -1.26621300 \\
\hline & 0.43891400 & 2.44152900 & -0.34868700 \\
\hline & 4.99136200 & 0.33475600 & -0.81197400 \\
\hline & 4. & .36108600 & 0.00055600 \\
\hline & 3.3 & 1.82521800 & 787100 \\
\hline & 2.52654800 & 1.14459300 & -0.80677100 \\
\hline & 2.81924200 & 0.06630800 & -1.62902900 \\
\hline & 4.16390000 & -0.42508900 & -1.66112500 \\
\hline & 1.69029300 & -0.36307600 & -2.29646700 \\
\hline & 4761100 & 0.44696800 & $-1 . \varepsilon$ \\
\hline
\end{tabular}




\begin{tabular}{|c|c|c|c|}
\hline $\mathrm{N}$ & 1.19111800 & 1.39092500 & -0.99012900 \\
\hline 0 & -5.64170900 & 1.49680200 & 0.67403800 \\
\hline & -4.87649700 & 0.53522300 & -1.65682100 \\
\hline H & -0.41648900 & -3.65236900 & -0.97286800 \\
\hline & -1.04067600 & -0.67380200 & 1.32247300 \\
\hline & -1.74023800 & -1.76049600 & -1.42614500 \\
\hline & -1.95257400 & -0.09915300 & -0.79818600 \\
\hline & -4.08314200 & -1.81097500 & -1.01362000 \\
\hline & -3.97419800 & -2.63640600 & 1.63607900 \\
\hline & -3.07299400 & -4.83664800 & 0.86904800 \\
\hline & -4.45741700 & -4.22981000 & -0.07904000 \\
\hline & -2.70106300 & -4.96178200 & -1.44087200 \\
\hline & -2.71085700 & 1.39981100 & 1.76838000 \\
\hline & -3.49887000 & 2.97083100 & 1.47259400 \\
\hline & -1.15716100 & 3.36887300 & 1.83678300 \\
\hline & -2.36086500 & 4.15504500 & -0.65 \\
\hline & -1.02825400 & 2.61811500 & -1.92110400 \\
\hline & 0.10322700 & 4.00343300 & -1.86 \\
\hline & 1.17639600 & 3.06723100 & 0.16787800 \\
\hline & -1.30887100 & 5.58934100 & 0.81 \\
\hline & -0.28155600 & 0.41928100 & -2.22449600 \\
\hline & 5.50271200 & 1.86256600 & 0.87198400 \\
\hline H & 6.48652000 & 1.76637400 & 0.65131600 \\
\hline $\mathrm{H}$ & 5.25311000 & 2.75717300 & 1.27 \\
\hline $\mathrm{H}$ & 5.94784300 & -0.00081800 & -0.74035800 \\
\hline 0 & 4.63265800 & -1.36551800 & -2.29 \\
\hline $\mathrm{N}$ & 3.19491900 & 0.41417800 & 2.95653900 \\
\hline $\mathrm{H}$ & 4.16261200 & 0.71069200 & 2.88 \\
\hline $\mathrm{H}$ & 2.54401000 & 1.19016700 & 2.99340900 \\
\hline $\mathrm{H}$ & 4.39380500 & -2.96023600 & 0.425 \\
\hline & & & \\
\hline & 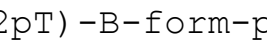 & & \\
\hline $\mathrm{C}$ & 2.39913300 & -4.31505900 & -0.50774000 \\
\hline O & 1.58177400 & -4.30875300 & 0.65581300 \\
\hline $\mathrm{C}$ & 2.48093600 & -2.90405400 & -1.05619200 \\
\hline O & 1.21772300 & -2.50004200 & -1.60257100 \\
\hline C & 2.84451400 & -1.85335300 & -0.00088500 \\
\hline O & 3.66015300 & -0.87261500 & -0.64129100 \\
\hline $\mathrm{C}$ & 1.48533300 & -1.29664300 & 0.41520300 \\
\hline $\mathrm{C}$ & 0.63924800 & -1.44334800 & -0.85146400 \\
\hline $\mathrm{N}$ & -4.34042600 & -0.42921700 & -1.82182300 \\
\hline $\mathrm{C}$ & -3.21283700 & 0.19631600 & -2.23860500 \\
\hline $\mathrm{N}$ & -1.95174600 & -0.11685100 & -1.91397400 \\
\hline C & -1.87008300 & -1.16988500 & -1.10769000 \\
\hline $\mathrm{C}$ & -2.95683000 & -1.91288200 & -0.61178300 \\
\hline C & -4.21519400 & -1.48014700 & -1.01437800 \\
\hline $\mathrm{N}$ & -2.50893000 & -2.94992800 & 0.19234700 \\
\hline $\mathrm{C}$ & -1.20927300 & -2.82937400 & 0.17452200 \\
\hline $\mathrm{N}$ & -0.75219600 & -1.76702300 & -0.58333300 \\
\hline $\mathrm{P}$ & 4.32781200 & 0.33774700 & 0.25531500 \\
\hline $\mathrm{C}$ & 2.72427500 & 2.00282100 & -0.96791500 \\
\hline O & 3.10420300 & 1.43690800 & 0.27885600 \\
\hline C & 1.42977100 & 2.75947000 & -0.79619400 \\
\hline O & 0.38683600 & 1.84160200 & -0.46627100 \\
\hline C & 1.42558000 & 3.84970200 & 0.31280000 \\
\hline O & 1.21914900 & 5.14688500 & -0.22966400 \\
\hline 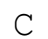 & 9021 & 19 & 1.162539 \\
\hline
\end{tabular}




\begin{tabular}{|c|c|c|c|}
\hline & -0.59746400 & 2.60187900 & 000 \\
\hline & -1.53867100 & 1.69192500 & 300 \\
\hline & -2.87968200 & 1.78643100 & 400 \\
\hline & -3.33841100 & 2.61814400 & -0.26158900 \\
\hline & -3.67846500 & 0.85733900 & 1.13725500 \\
\hline & -3.29783000 & -0.13986800 & 2.032434 \\
\hline & -4.14113800 & -0.88268800 & 2.518485 \\
\hline & -1.86748900 & -0.18793300 & 2.31512200 \\
\hline & -1.07816600 & 0.72514400 & 1.71104500 \\
\hline & -1.36308000 & -1.21897100 & 3.27863500 \\
\hline & 5.45686300 & 0.87885300 & -0.57057300 \\
\hline & 4.4997740 & -0.11842800 & 1.67314 \\
\hline & -0.50171100 & -3.4871 & 0.6665 \\
\hline & 0.61992700 & -0.52482000 & -1.44525700 \\
\hline & 1.07438300 & -1.90735300 & 1.22377600 \\
\hline & 1.55049100 & -0.25549600 & 0.73277300 \\
\hline & 3.38943800 & -2.28546900 & 0.84248500 \\
\hline & 3.2 & -2.88763500 & -1 \\
\hline & 1.97799000 & -4.97427500 & -1.27632200 \\
\hline & 3.41407600 & -4.65627900 & -0.26614000 \\
\hline & 1.53841600 & -5.20670800 & 1.01539100 \\
\hline & 2.58795300 & 1.21276500 & -1.71769100 \\
\hline & 3.5 & 2. & -1 \\
\hline & 1.18398500 & 3.25178800 & -1.74 \\
\hline & 2.35220500 & 3.82593500 & 0.89452900 \\
\hline & 0.51482600 & 2.90614700 & 2.03945100 \\
\hline & -0.33812400 & 4.36986400 & 1.49159600 \\
\hline & -1.17635900 & $U \perp 6 \perp 6$ & -0 . \\
\hline & 2. & 00 & -0 . \\
\hline & -4.66728900 & 3200 & 0700 \\
\hline & -1.63174700 & -2.22557000 & 2.94314900 \\
\hline & -0.27620800 & -1.15799600 & 3.37679000 \\
\hline & -1.80841300 & -1.07411300 & 350300 \\
\hline & -0.0 & 0. & 0 \\
\hline & -3.38813 & 47300 & 5600 \\
\hline & -4.29895300 & 1.66852100 & -3.07958500 \\
\hline & -2.62356100 & 1.89311000 & -3.15218000 \\
\hline & -5.12950100 & -1.97046400 & -0.68616100 \\
\hline \multirow{2}{*}{\multicolumn{4}{|c|}{$\begin{array}{l}63 \\
d(\mathrm{ApA})-\mathrm{B}-\mathrm{form}-\mathrm{pcm}\end{array}$}} \\
\hline & & & \\
\hline & 2.70537500 & -4.35252200 & 0.07258900 \\
\hline & 1.80875100 & -4.21663000 & 1.16765500 \\
\hline & 2.83134600 & -3.01347100 & -0.62729500 \\
\hline & 1.602329 & -2.67079500 & -1.28236000 \\
\hline & 3.1 & -1.84836500 & 0.31713800 \\
\hline & 3.982611 & -0.93758900 & -0.39749600 \\
\hline & 1.769961 & -1.25862300 & 0.60654100 \\
\hline & 0.99578500 & -1.53501600 & -0.68296000 \\
\hline & -3.85265900 & -0.46298400 & -2.06522600 \\
\hline & -2.679333 & 0.06176700 & -2.45477900 \\
\hline & -1.45506500 & -0.25647300 & -2.04957500 \\
\hline & -1.47392500 & -1.23118400 & -1.13014500 \\
\hline & -2.60579100 & -1.88252700 & -0.64221700 \\
\hline & -3.84371700 & -1.44499300 & -1.15309700 \\
\hline & -2.26271300 & -2.84762500 & 0.28341300 \\
\hline & -0 . & -2 & \\
\hline & -0.4151890 & -1.81010600 & -0.4709670 \\
\hline
\end{tabular}




\begin{tabular}{|c|c|c|c|}
\hline P & 4.60652200 & 0.37420500 & 0.3795500 \\
\hline C & 3.04295200 & 1.90195700 & -1.0616710 \\
\hline & 3.37276500 & 1.45370200 & 0 \\
\hline & 1.75547300 & 2.68959100 & -1.00 \\
\hline & 0.67938700 & 1.80816800 & -0.67254 \\
\hline & 1.72916100 & 3.84057200 & \\
\hline & 1.36935100 & 5.08079900 & -0.5 \\
\hline & 0.59883700 & 3.43 & \\
\hline & -0.26509900 & 2.5710400 & \\
\hline & -5.16 & 1.12 & \\
\hline & -4.60 & 2.06 & -0 \\
\hline & -3.31 & 2.39 & -0.2 \\
\hline & -2.54 & 1.63 & \\
\hline & -2.98 & 0.62 & \\
\hline & -4.37 & $0.3 \xi$ & \\
\hline & -4.92 & -0.5 & \\
\hline & -1.93 & 0.0 & \\
\hline & -0 . & 0.71 & \\
\hline & -1.18 & 1.68 & 0 \\
\hline & 5.75 & 0.8 & -0 \\
\hline & 4.73 & $0.0^{\circ}$ & 1. \\
\hline $\mathrm{H}$ & -0.31 & $-3 \cdot 3$ & \\
\hline & 1.02 & $-0.6 \varepsilon$ & -1 \\
\hline & 1.31 & $-1.7 \xi$ & \\
\hline & 1.82 & $-0.1 \xi$ & 0 . \\
\hline & 3.65 & -2.1 & 1 . \\
\hline & 3.61 & -3.09 & $-1 \cdot 3$ \\
\hline I & 2.33 & -5.0 & -0 \\
\hline & 3.69 & -4.66 & 0. \\
\hline $\mathrm{H}$ & 1.72 & $-5.0^{\circ}$ & \\
\hline & 2.91 & 1.04 & -1 \\
\hline $\mathrm{H}$ & 3.84 & 2.5 & -1 \\
\hline & 1.56 & 3.12 & -1 \\
\hline $\mathrm{H}$ & 2 . & 3.9 & \\
\hline & 0.98 & 2.81 & 1 . \\
\hline $\mathrm{H}$ & 0 . & 4.2 & 1 . \\
\hline & -0.87 & 3.18 & -0.6 \\
\hline $\mathrm{H}$ & 2.09 & $5.3 \xi$ & -1 \\
\hline & -5.29 & 2.63 & -0.7 \\
\hline $\mathrm{H}$ & -4.33 & -1.22 & 2 . \\
\hline & -5.90 & -0.7 & 2. \\
\hline $\mathrm{H}$ & 0 . & 0 . & \\
\hline & -2.74 & 0.85 & -3 \\
\hline N & -5 & -2 & -0 \\
\hline & -5 & -1 & -0 \\
\hline$\Pi$ & -5.02 & -2.5 & \\
\hline 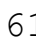 & & & \\
\hline & $\sigma$ & & \\
\hline C & 1. & -4.5423440 & -0 \\
\hline 0 & 1. & -4.34 & 1 \\
\hline $\mathrm{C}$ & 2.17171900 & -3.2 & -0 \\
\hline O & 0.97247400 & -2 & -1 \\
\hline C & & -2.1 & \\
\hline O & & -1 & -0 \\
\hline C & & -1 & \\
\hline & & -1 & -0 \\
\hline N & -4 & 0 & -1 \\
\hline
\end{tabular}




\begin{tabular}{|c|c|c|c|}
\hline & -3.06689600 & 0.45025900 & -2.32285600 \\
\hline & -1.86102500 & 0.022 & -1.9 \\
\hline & -1.93509100 & -0.96255900 & -1.05 \\
\hline & -3.10525300 & -1.50072700 & -0.51948700 \\
\hline & -4.31418900 & -0.93975600 & -0.97228800 \\
\hline & -2.82117300 & -2.50884200 & 0.38133500 \\
\hline & -1.51221400 & -2.573056 & 0.381 \\
\hline & -0.91318800 & -1.66137100 & -0.456770 \\
\hline & 4.37054400 & -0.09479000 & 0.31366800 \\
\hline & 2.96861800 & 1.65466400 & -1.03417200 \\
\hline & 3.27955400 & 1.13438200 & 0.25115500 \\
\hline & 1.77382500 & 2.57077600 & 93600 \\
\hline & 0.62986200 & 1.81134100 & -0.54079800 \\
\hline & 1.89874800 & 3.73080000 & 0.10174700 \\
\hline & 1.89207400 & 5.00038300 & -0.53746700 \\
\hline & 0.62430800 & 3.6003480 & 0.93619100 \\
\hline & -0.26961900 & 2.73949500 & 0.04158600 \\
\hline & -1.31821300 & 00400 & 422100 \\
\hline & -2.67309800 & 2.27426500 & 0.42437700 \\
\hline & -2.94832600 & 3.20391100 & -0.33734900 \\
\hline & -3.62078900 & 1.48856100 & 1.00332900 \\
\hline & -3.27644200 & 0.51131600 & 1.82806800 \\
\hline & -1.91693600 & 0.2 & 2 . \\
\hline & -0.97814600 & 1.02844100 & 1.61321400 \\
\hline & 5.54945200 & 0.25270800 & -0.54626000 \\
\hline & 4.49628900 & -0.45933900 & 1.76261400 \\
\hline & -0.90912300 & -3.27341300 & 0.94746400 \\
\hline & 0.6 & -0.666600 & -1.38212400 \\
\hline & $0 . \varepsilon$ & -1 . & 5500 \\
\hline & 1.53586800 & -0.32439200 & 392400 \\
\hline & 3.12773500 & -2.53273300 & 1.09842800 \\
\hline & 2.91663600 & -3.34260900 & -1.54853100 \\
\hline & 1.43675100 & -5.21307100 & -0.81997200 \\
\hline & 2.8 & -4 & 4600 \\
\hline & 0.90376800 & -5.19697600 & 408800 \\
\hline & 2.73830600 & 0.83655500 & -1.72894200 \\
\hline & 3.82345600 & 2.21565500 & -1.43107200 \\
\hline & 1.58965300 & 3.02066200 & -1.91361100 \\
\hline & 2.7 & 3.6211 & 0.71315000 \\
\hline & 0.8 & 0 & 00 \\
\hline & 0.18818400 & 4.57454600 & 1.16683500 \\
\hline & -0.76391800 & 3.33608800 & -0.72934100 \\
\hline & 2.72546600 & 5.11689700 & -1.01907100 \\
\hline & 0.08386500 & 0.90735 & 1.79542000 \\
\hline & -4.26719700 & -0.25019300 & 2.34391000 \\
\hline & -5.159815 & -0.21222200 & 1.86302100 \\
\hline & -4.02301200 & -1.14985400 & 2.73816000 \\
\hline & -1.65036400 & -0.52501200 & 2.90865500 \\
\hline & -3.08883800 & 1.24478200 & -3.06456700 \\
\hline & -5.51818600 & -1.31592300 & -0.46640100 \\
\hline & -6.33951000 & -1.05013800 & -0.99596400 \\
\hline & -5.56903200 & -2.20875300 & 0.00888700 \\
\hline \multirow{2}{*}{\multicolumn{4}{|c|}{$\begin{array}{l}4 \\
(\mathrm{Apg})-\mathrm{B}-\text { form-pcm }\end{array}$}} \\
\hline & & & \\
\hline & 3.18812200 & -4.18935700 & -0.00368000 \\
\hline & 2.315153 & -4.12936800 & 1.11681700 \\
\hline & 3.188546 & -2.84170800 & -0.69843100 \\
\hline
\end{tabular}




\begin{tabular}{|c|c|c|c|}
\hline & 1.92070700 & -2.59614200 & -1.3223380 \\
\hline & 3.43225400 & -1.65820700 & 0.24 \\
\hline & 4.17462500 & -0.68153600 & \\
\hline & 2.01984100 & -1.18174500 & \\
\hline & 1.23877200 & -1.51595400 & \\
\hline & 954600 & -0.82870100 & -1 \\
\hline & -2.60464800 & -0.20693600 & $-2 \cdot 3$ \\
\hline & -1.3477 & -0.42609700 & -1.9 \\
\hline & -1.2599380 & -1.40891400 & \\
\hline & -2.31 & -2.15788800 & -0 \\
\hline & -3.6037140 & -1.81624500 & $-1 \cdot 0$ \\
\hline & -1.87 & -3.10 & \\
\hline & -0.57 & -2.92105100 & 0.3 \\
\hline & -0.13 & -1.907769 & -0.4 \\
\hline & 4.72808600 & 0.66396600 & \\
\hline & 8900 & 2 . & $-1 \cdot 0$ \\
\hline & 3.41 & 1.64895 & \\
\hline & 1.66 & 2.77 & \\
\hline & 0.66 & 1.80 & -0 \\
\hline & 1.57 & 3.8 & \\
\hline & 1.07 & 5.10 & -0 \\
\hline & 0.5 & 3.35 & \\
\hline & -0.29 & 2.46 & 0.1 \\
\hline & -4.9 & 0.6 & \\
\hline & -4.5 & 1.67 & -0 \\
\hline & -3.2 & 2.0 & -0.1 \\
\hline & -2.47 & 1.32 & 0 . \\
\hline & -2.82 & 0.2 & \\
\hline & -4.19 & -0.12 & 1.5 \\
\hline & -1.72 & -0.1 & \\
\hline & -0.73 & 0.5 & 1. \\
\hline $\mathrm{N}$ & -1.12 & 1.4 & \\
\hline & 5.80 & 1.22 & -0 \\
\hline 0 & 4.93 & 0.3 & \\
\hline & 0.13 & -3.4 & 0.9 \\
\hline $\mathrm{H}$ & $1.1 \xi$ & -0.6 & -1 \\
\hline & 1.62 & -1.7 & 1 \\
\hline $\mathrm{H}$ & 1.9 & -0.1 & \\
\hline & 3.9 & -1.9 & 1.1 \\
\hline $\mathrm{H}$ & 3.9 & -2.8 & -1 \\
\hline & 2.85 & -4.9 & -0.7 \\
\hline$H$ & 4.2 & -4.4 & \\
\hline & 2.31 & -4.9 & 1.5 \\
\hline H & 2.9 & 1.2 & -1. \\
\hline & 3.73 & 2.8 & $-1 \cdot 4$ \\
\hline $\mathrm{H}$ & 1.4 & 3.2 & -1. \\
\hline & 2.54 & 4.05 & \\
\hline $\mathrm{H}$ & 1.0 & 2.7 & \\
\hline & -0.03 & 4.1 & 1.5 \\
\hline $\mathrm{H}$ & -0.97 & 3.0 & -0. \\
\hline $\mathrm{H}$ & 1.74 & 5.4 & -1 \\
\hline $\mathrm{H}$ & 0.29 & 0.4 & \\
\hline & -5.44 & 2.2 & -0 \\
\hline $\mathrm{H}$ & -6.42 & 2.24 & -0 \\
\hline & -5.14 & 3.1 & -1 \\
\hline 11 & -5 & 0.4 & 0 \\
\hline & - & $-1 \cdot 0$ & 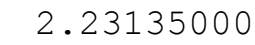 \\
\hline
\end{tabular}




\begin{tabular}{|c|c|c|c|}
\hline & -2.76038400 & 0.58194500 & -3.08280300 \\
\hline & -4.72113500 & -2.47631900 & -0.63125100 \\
\hline & -5.60473900 & -2.02455300 & 010 \\
\hline & -4.67860300 & -2.95952000 & 0.25797600 \\
\hline & & & \\
\hline & $\mathrm{DT})-\mathrm{B}-$ & & \\
\hline & 2.26628500 & -4.40851800 & -0.18463200 \\
\hline & 1.36160700 & -4.26159300 & 0200 \\
\hline & 2.48160700 & -3.05401100 & -0.83129400 \\
\hline & 1.28044000 & -2.61310800 & -1.48017900 \\
\hline & 2.85904600 & -1.94497700 & 0.15760400 \\
\hline & 3.74129300 & -1.05250600 & -0.52356700 \\
\hline & 1.51484100 & -1.29425800 & \\
\hline & 0.74813600 & -1.45972000 & $-0 . \varepsilon$ \\
\hline & -4.02632100 & 3300 & -2.176985 \\
\hline & -2.82136800 & 0.40729400 & -2.60963800 \\
\hline & -1.61813800 & -0.00342000 & -2.22315600 \\
\hline & -1.69752200 & -0.97115700 & -1.29675600 \\
\hline & -2.87071600 & -1.52 & 9100 \\
\hline & -4.07853700 & -0.96165400 & -1.2423280 \\
\hline & -2.59382500 & -2.51673000 & 0.134608 \\
\hline & -1.28558000 & -2.55652400 & 0.17186000 \\
\hline & -0.68083100 & -1.63804400 & -0.65623300 \\
\hline & 4.453 & 0.17822100 & \\
\hline & 2.89856600 & 4100 & -0 . \\
\hline & 3.26494200 & 1.31563800 & 0.29621500 \\
\hline & 1.61488000 & 2.63744500 & -0.82781500 \\
\hline & 0.54317600 & 1.75865500 & -0.48774700 \\
\hline & 1.6 & 500 & \\
\hline & 1.67656300 & 5. & -0 \\
\hline & 0.23319800 & 3.58628300 & 38500 \\
\hline & -0.48492900 & 2.61239700 & -0.02487500 \\
\hline & -1.52494000 & 1.80636900 & 0.59451000 \\
\hline & -2.84091600 & 8500 & 848600 \\
\hline & -3.18924200 & 0400 & -0 . \\
\hline & -3.748 & 1.226 & 27800 \\
\hline & -3.48932100 & 0.21597400 & 1.80410400 \\
\hline & -4.41127200 & -0.45690000 & 2.25063800 \\
\hline & -2.08310700 & 0.06280400 & 2.16228100 \\
\hline & -1.187152 & 0.8587 & 00 \\
\hline & -1.7178 & -0.9 & \\
\hline & 5.58745800 & 300 & -0.55623300 \\
\hline & 4.62880000 & -0.21574800 & 1.74374000 \\
\hline & -0.68555300 & -3.24009800 & 0.76125100 \\
\hline & 0.85089200 & -0.58948700 & -1.50108400 \\
\hline & 1.0 & -1.84963300 & 1.26823500 \\
\hline & $1.612^{\circ}$ & -0.24470000 & 55400 \\
\hline & 3.35195100 & -2.33573300 & 192700 \\
\hline & 3.26181800 & -3.15258100 & -1.59293900 \\
\hline & 1.86410400 & -5.10043000 & -0.93437700 \\
\hline & 3.23479800 & -4.78955900 & 0.16457700 \\
\hline & 1.20743600 & -5.12951800 & 1.30278800 \\
\hline & 2.75757300 & 1.05096600 & -1.69839600 \\
\hline & 3.68848000 & 2.52143000 & -1.33425000 \\
\hline & 1.39424700 & 3.11021900 & -1.79786400 \\
\hline & 2.4178540 & 3.62060100 & 0.96217200 \\
\hline & 0.33742900 & 3.13355600 & 1.90489700 \\
\hline
\end{tabular}




\begin{tabular}{|c|c|c|c|}
\hline $\mathrm{H}$ & -0.28972500 & 4.53980000 & 1.01445300 \\
\hline $\mathrm{H}$ & -0.95688900 & 3.13240500 & -0.86353900 \\
\hline $\mathrm{H}$ & 2.58221700 & 5.20777300 & -0.64617900 \\
\hline $\mathrm{H}$ & -4.72089000 & 1.37101000 & 0.62133700 \\
\hline & -2.08790700 & -1.94787600 & 2.89871000 \\
\hline $\mathrm{H}$ & -0.63334700 & -1.01406300 & 3.31906600 \\
\hline & -2.16802500 & -0.71544300 & 4.16067600 \\
\hline 4 & -0.12292700 & 0.79283400 & 1.74498600 \\
\hline & -2.84075100 & 1.18558400 & -3.36837200 \\
\hline 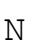 & -5.28369000 & -1.37719400 & -0.78147400 \\
\hline & -6.08194100 & -0.79204400 & -0.99568600 \\
\hline & -5.30385000 & -1.84079600 & 0.11933900 \\
\hline & & & \\
\hline & A) -Twistec & $\mathrm{cm}$ & \\
\hline $\mathrm{C}$ & 3.588088 & -1.002591 & -1.656884 \\
\hline C & 2.508030 & -0.129794 & -1.420635 \\
\hline $\mathrm{C}$ & 2.814503 & 1.071836 & -0.784704 \\
\hline $\mathrm{N}$ & 4.031613 & 1.476562 & -0.394564 \\
\hline $\mathrm{C}$ & 4.964768 & 0.576502 & -0.689504 \\
\hline $\mathrm{N}$ & 4.818574 & -0.615206 & -1.290512 \\
\hline $\mathrm{N}$ & 1.613138 & 1.726994 & -0.654397 \\
\hline $\mathrm{C}$ & 0.663484 & 0.894803 & -1.200742 \\
\hline $\mathrm{N}$ & 1.153937 & -0.224731 & -1.674153 \\
\hline C & 1.392451 & 2.979540 & 0.062247 \\
\hline O & 0.563142 & 2.731693 & 1.182363 \\
\hline $\mathrm{C}$ & -0.702948 & 3.399597 & 1.063166 \\
\hline C & -0.781026 & 3.892873 & -0.390728 \\
\hline $\mathrm{C}$ & 0.688905 & 4.060150 & -0.759518 \\
\hline C & -1.821678 & 2.461971 & 1.480533 \\
\hline 0 & -1.992483 & 1.424252 & 0.523910 \\
\hline $\mathrm{P}$ & -3.354805 & 1.294613 & -0.398867 \\
\hline O & -2.909589 & 1.267666 & -1.834684 \\
\hline O & -1.461373 & 5.127409 & -0.517328 \\
\hline $\mathrm{N}$ & 3.425996 & -2.204271 & -2.266396 \\
\hline O & -3.868603 & -0.194504 & 0.057147 \\
\hline $\mathrm{C}$ & -3.438357 & -1.412823 & -0.562345 \\
\hline C & -3.634832 & -2.522074 & 0.484701 \\
\hline 0 & -2.402118 & -2.580981 & 1.221223 \\
\hline C & -1.435714 & -1.728127 & 0.623038 \\
\hline $\mathrm{C}$ & -1.936767 & -1.467358 & -0.789381 \\
\hline C & -3.959860 & -3.891114 & -0.082001 \\
\hline 0 & -2.955263 & -4.263766 & -1.015347 \\
\hline $\mathrm{N}$ & -0.133928 & -2.355824 & 0.673530 \\
\hline $\mathrm{C}$ & 0.253949 & -3.557390 & 0.117890 \\
\hline $\mathrm{N}$ & 1.537232 & -3.793224 & 0.211830 \\
\hline C & 2.044481 & -2.670552 & 0.855809 \\
\hline C & 1.009105 & -1.765641 & 1.139615 \\
\hline $\mathrm{N}$ & 1.138071 & -0.573501 & 1.705070 \\
\hline C & 2.408574 & -0.272491 & 1.999093 \\
\hline $\mathrm{N}$ & 3.494558 & -1.060038 & 1.803885 \\
\hline $\mathrm{C}$ & 3.316512 & -2.250827 & 1.234539 \\
\hline $\mathrm{N}$ & 2.628461 & 0.947604 & 2.591123 \\
\hline O & -4.373557 & 2.278793 & 0.094199 \\
\hline $\mathrm{H}$ & -3.156723 & -5.148028 & -1.353966 \\
\hline $\mathrm{H}$ & 4.203238 & -2.859846 & 1.067557 \\
\hline $\mathrm{H}$ & -4.946403 & -3.849543 & -0.562208 \\
\hline $\mathrm{H}$ & -4.003218 & -4.609152 & 0.746112 \\
\hline
\end{tabular}




$$
\begin{array}{r}
-4.435551 \\
-4.021435 \\
-1.702820 \\
-1.528774 \\
-1.342229 \\
-0.484807 \\
1.888504 \\
3.565166 \\
5.982758 \\
-2.750312 \\
-1.572717 \\
-0.701889 \\
-1.260830 \\
-2.415769 \\
0.892211 \\
1.021982 \\
2.383922 \\
-0.386422 \\
4.190795 \\
2.498570
\end{array}
$$

$-2.236329$ $-1.584306$ $-2.321230$ $-0.562766$ $-0.798848$

$-4.212436$ 1.625406

1.311830

0.832882

3.031922

2.002439

4.271501

3.136240

4. 959801

3.972793

5.044594

3.286730

1.167862

$-2.862206$

$-2.612493$

61

$\mathrm{d}(2 \mathrm{pc})-$ Twisted-pcm

C

2.506108

1.205116

0.437082

0.815235

2.091871

2.943237

$-0.759780$

$-0.659486$

0.483391

$-1.721437$

$-2.548999$

$-3.704637$

$-3.775782$

$-2.699577$

$-3.640075$

$-2.238153$

$-2.588451$

$-4.898826$

$-5.055285$

2.576305

$-1.560631$

$-1.270005$

0.110368

1.114958

2.263868

1.926453

0.403681

2.578356

3.914005

4.187293

3.224891

1.871964

1.598942

4.783389

3.561667
$-3.526766$

$-3.485607$

$-2.402698$

$-1.448813$

$-1.575166$

$-2.575313$

$-2.492382$

$-3.614470$

$-4.238229$

$-1.418316$

$-1.558789$

$-0.728513$

$-0.394220$

$-1.304015$

1.015040

1.850341

3. 315140

$-1.519920$

$-2.725062$

$-0.613072$

1.470134

2. 443233

3.034440

2.030807

2. 346075

3. 628222

3.593323

1.185539

0.727122

$-0.372153$

$-0.976706$

$-0.510045$

0.560626

1. 332743

$-2.063264$
1.174818

$-1.472067$

$-1.430371$

$-1.230472$

1.192224

$-0.330674$

2.423042

2.462026

$-0.406652$

1.590306

2.442986

1.731332

$-1.023122$

$-0.547784$

$-1.828107$

$-0.413871$

0.403887

$-1.201794$

$-2.175657$

$-2.249462$

0.576378

0.077283

0.520259

1.358763

1.739242

1.397907

$-0.145664$

$-0.947058$

$-0.842433$

$-0.109754$

1.039630

0.856513

$-0.657080$

$-1.268657$

$-0.886880$

$-0.953725$

$-0.958692$

1.359466

0.628481

2.588178

0.495895

1. 487511

1.293892

1.479421

0.704958

$-0.078382$

$-0.100674$

$-0.146956$

$-0.200219$

$-0.951052$

$-1.636360$

$-1.642937$

$-0.857346$

0.436131

$-2.358679$ 


\begin{tabular}{|c|c|c|c|}
\hline 0 & -0.211026 & 4.835084 & -0.343220 \\
\hline 0 & -1.345688 & 1.265856 & -2.011135 \\
\hline $\mathrm{H}$ & -4.234564 & -3.232466 & 0.739197 \\
\hline $\mathrm{H}$ & 3.209140 & -4.312141 & 0.306714 \\
\hline $\mathrm{H}$ & -5.814301 & -0.939057 & 1.219113 \\
\hline $\mathrm{H}$ & -4.777462 & -1.727135 & 2.430287 \\
\hline $\mathrm{H}$ & -3.592453 & 0.199860 & 1.427730 \\
\hline $\mathrm{H}$ & -4.761044 & -0.636074 & -1.059223 \\
\hline $\mathrm{H}$ & -3.161869 & -2.276112 & -1.471125 \\
\hline $\mathrm{H}$ & -2.249913 & -0.896136 & -2.173345 \\
\hline $\mathrm{H}$ & -1.125713 & -0.510393 & -0.009112 \\
\hline $\mathrm{H}$ & -1.471723 & -3.914495 & -1.596161 \\
\hline $\mathrm{H}$ & 2.067082 & 0.266358 & 2.566493 \\
\hline $\mathrm{H}$ & 3.584603 & -0.526438 & 2.622314 \\
\hline $\mathrm{H}$ & -2.006943 & 3.253354 & 1.464111 \\
\hline $\mathrm{H}$ & -1.328283 & 1.936996 & 2.455420 \\
\hline $\mathrm{H}$ & 0.248715 & 3.839316 & 2.032292 \\
\hline $\mathrm{H}$ & 0.082039 & 2.876769 & -0.862798 \\
\hline $\mathrm{H}$ & -1.128501 & 4.616481 & -0.612813 \\
\hline $\mathrm{H}$ & 2.388658 & 3.638744 & -1.067541 \\
\hline $\mathrm{H}$ & 2.267105 & 4.504126 & 0.484463 \\
\hline $\mathrm{H}$ & 3.130259 & 2.468262 & 1.352549 \\
\hline $\mathrm{H}$ & 0.586945 & 0.939269 & -0.751399 \\
\hline $\mathrm{H}$ & 1.089120 & -1.007139 & -2.204824 \\
\hline $\mathrm{H}$ & 4.487950 & -2.454328 & -2.248752 \\
\hline $\mathrm{H}$ & 2.850372 & -2.646153 & -2.777422 \\
\hline \multicolumn{4}{|c|}{64} \\
\hline \multicolumn{4}{|c|}{$d(2 p G)-T w i s t e d-p c m$} \\
\hline $\mathrm{C}$ & 3.316754 & -1.289517 & -1.747046 \\
\hline $\mathrm{C}$ & 2.323308 & -0.297378 & -1.466332 \\
\hline $\mathrm{C}$ & 2.700715 & 0.841830 & -0.767168 \\
\hline $\mathrm{N}$ & 3.922492 & 1.164702 & -0.279078 \\
\hline $\mathrm{C}$ & 4.825978 & 0.242571 & -0.516367 \\
\hline $\mathrm{N}$ & 4.565112 & -0.906416 & -1.218911 \\
\hline $\mathrm{N}$ & 1.564760 & 1.600937 & -0.667747 \\
\hline $\mathrm{C}$ & 0.564773 & 0.882686 & -1.293219 \\
\hline $\mathrm{N}$ & 0.982028 & -0.254709 & -1.786110 \\
\hline $\mathrm{C}$ & 1.440101 & 2.853401 & 0.072414 \\
\hline O & 0.618310 & 2.647142 & 1.207159 \\
\hline $\mathrm{C}$ & -0.604597 & 3.393753 & 1.119133 \\
\hline $\mathrm{C}$ & -0.677728 & 3.906262 & -0.327580 \\
\hline $\mathrm{C}$ & 0.793128 & 3.991318 & -0.719559 \\
\hline $\mathrm{C}$ & -1.770753 & 2.519573 & 1.543419 \\
\hline 0 & -2.023712 & 1.513088 & 0.572808 \\
\hline $\mathrm{P}$ & -3.352912 & 1.549980 & -0.402492 \\
\hline 0 & -2.861044 & 1.490491 & -1.822540 \\
\hline 0 & -1.298881 & 5.173626 & -0.430468 \\
\hline $\mathrm{N}$ & 6.083897 & 0.383354 & -0.015209 \\
\hline O & 3.208863 & -2.353015 & -2.351767 \\
\hline 0 & -4.045365 & 0.122903 & 0.012264 \\
\hline C & -3.704639 & -1.130137 & -0.595564 \\
\hline $\mathrm{C}$ & -4.037926 & -2.222582 & 0.435893 \\
\hline O & -2.835578 & -2.421006 & 1.198904 \\
\hline $\mathrm{C}$ & -1.775609 & -1.658475 & 0.642512 \\
\hline C & -2.208198 & -1.325383 & -0.776717 \\
\hline $\mathrm{C}$ & -4.489882 & -3.544104 & -0.155384 \\
\hline 0 & -3.505929 & -4.006816 & 9896 \\
\hline
\end{tabular}




\begin{tabular}{|c|c|c|c|}
\hline J & -0.541466 & -2.411904 & 0.704864 \\
\hline & -0.271637 & -3.654289 & 0.167570 \\
\hline & 0.987618 & -3.998750 & 0.238951 \\
\hline & 1.606986 & -2.908535 & 0.838850 \\
\hline & 0.659246 & -1.913604 & 1.130725 \\
\hline & 0.900243 & -0.733548 & 1.683366 \\
\hline & 2.196932 & -0.538072 & 1.949949 \\
\hline & 3.210896 & -1.407923 & 1.720112 \\
\hline & 2.919702 & -2.586612 & 1.169511 \\
\hline & 2.526795 & 0.653574 & 2.550417 \\
\hline & -4.268979 & 2.643010 & 0.063384 \\
\hline & -3.791316 & -4.858330 & -1.431354 \\
\hline & 3.749781 & -3.265351 & 0.981417 \\
\hline & -5.454788 & -3.394908 & -0.657645 \\
\hline & -4.627279 & -4.263196 & 0.661491 \\
\hline & -4.819842 & -1.861223 & 1.111961 \\
\hline & -4.270457 & -1.245595 & -1.524836 \\
\hline & -2.038784 & -2.188536 & -1.426114 \\
\hline & -1.695349 & -0.459588 & -1.185452 \\
\hline & -1.602913 & -0.752965 & 1.231275 \\
\hline & -1.074983 & -4.249729 & -0.252136 \\
\hline & 1.839161 & 1.388261 & 2.397988 \\
\hline & 3.482901 & 0.947587 & 2.385195 \\
\hline & -2.659934 & 3.142596 & 1.685525 \\
\hline & -1.529037 & 2.023180 & 2.489149 \\
\hline & -0.537894 & 4.255984 & 1.796627 \\
\hline & -1.205164 & 3.184563 & -0.961277 \\
\hline & -2.260485 & 5.045741 & -0.439899 \\
\hline & 0.972205 & 3.910588 & -1.793103 \\
\hline & 1.194626 & 4.946006 & -0.363243 \\
\hline & 2.454705 & 3.089464 & 0.400841 \\
\hline & -0.455065 & 1.249850 & -1.342052 \\
\hline & 5.307515 & -1.588276 & -1.346081 \\
\hline & 6.309273 & 1.317049 & 0.305450 \\
\hline & 6.848754 & -0.099725 & -0.469962 \\
\hline \multirow{2}{*}{\multicolumn{4}{|c|}{$\begin{array}{l}63 \\
d(2 p T)-T w i s t e d-p c m\end{array}$}} \\
\hline & & & \\
\hline $\mathrm{C}$ & -1.674802 & 0.713257 & 0.900724 \\
\hline J & -2.564603 & 1.366606 & 0.066602 \\
\hline$C$ & -3.860832 & 0.913393 & -0.093696 \\
\hline $\mathrm{J}$ & -4.171715 & -0.195409 & 0.661502 \\
\hline C & -3.347691 & -0.892818 & 1.542151 \\
\hline C & -1.996337 & -0.363779 & 1.650190 \\
\hline C & -2.136252 & 2.538828 & -0.713263 \\
\hline 0 & -1.004792 & 2.164600 & -1.485317 \\
\hline C & 0.048475 & 3.114673 & -1.292744 \\
\hline C & -0.194452 & 3.654330 & 0.118919 \\
\hline C & -1.714059 & 3.751136 & 0.138337 \\
\hline C & 1.397058 & 2.462885 & -1.519883 \\
\hline 0 & 1.664439 & 1.460952 & -0.551001 \\
\hline P & 2.362985 & 1.796453 & 0.898333 \\
\hline 0 & 1.457043 & 1.235822 & 1.958568 \\
\hline ○ & 0.471456 & 4.866892 & 0.373248 \\
\hline 0 & -4.681860 & 1.432260 & -0.840634 \\
\hline 0 & -3.780449 & -1.870676 & 2.142250 \\
\hline C & -1.019112 & -1.055111 & 2.553144 \\
\hline o & 3.731548 & 0.912473 & 0.810163 \\
\hline
\end{tabular}




\begin{tabular}{|c|c|c|c|}
\hline $\mathrm{C}$ & 3.817080 & -0.492786 & 0.538458 \\
\hline C & 3.682778 & -0.795486 & -0.974154 \\
\hline 0 & 2.434045 & -1.472050 & -1.153902 \\
\hline $\mathrm{C}$ & 1.690526 & -1.409008 & 0.053859 \\
\hline $\mathrm{C}$ & 2.735508 & -1.385424 & 1.159499 \\
\hline $\mathrm{N}$ & 0.733031 & -2.492183 & 0.078386 \\
\hline $\mathrm{C}$ & -0.487940 & -2.386099 & -0.536259 \\
\hline $\mathrm{C}$ & -1.218609 & -3.512786 & -0.137810 \\
\hline $\mathrm{N}$ & -0.446502 & -4.309486 & 0.698909 \\
\hline $\mathrm{C}$ & 0.688789 & -3.669322 & 0.799021 \\
\hline $\mathrm{N}$ & -0.907507 & -1.405759 & -1.324383 \\
\hline $\mathrm{C}$ & -2.177747 & -1.563126 & -1.711204 \\
\hline $\mathrm{N}$ & -3.004354 & -2.588878 & -1.384912 \\
\hline $\mathrm{C}$ & -2.530807 & -3.559258 & -0.606170 \\
\hline $\mathrm{N}$ & -2.684449 & -0.607030 & -2.553042 \\
\hline $\mathrm{C}$ & 4.796946 & -1.678348 & -1.498210 \\
\hline 0 & 4.894463 & -2.817214 & -0.655005 \\
\hline 0 & 2.771517 & 3.246572 & 0.923268 \\
\hline $\mathrm{H}$ & 5.624410 & -3.374238 & -0.960405 \\
\hline $\mathrm{H}$ & -3.211932 & -4.368361 & -0.351698 \\
\hline $\mathrm{H}$ & 5.732901 & -1.104235 & -1.491692 \\
\hline $\mathrm{H}$ & 4.570790 & -1.972407 & -2.530563 \\
\hline $\mathrm{H}$ & 3.671290 & 0.146383 & -1.533968 \\
\hline $\mathrm{H}$ & 4.806770 & -0.773394 & 0.901099 \\
\hline $\mathrm{H}$ & 3.172984 & -2.380521 & 1.284868 \\
\hline $\mathrm{H}$ & 2.351720 & -1.007660 & 2.106920 \\
\hline $\mathrm{H}$ & 1.084468 & -0.500641 & 0.061562 \\
\hline $\mathrm{H}$ & 1.531938 & -3.996458 & 1.393002 \\
\hline $\mathrm{H}$ & -2.183448 & 0.275795 & -2.556681 \\
\hline $\mathrm{H}$ & -3.693087 & -0.539046 & -2.604514 \\
\hline $\mathrm{H}$ & 2.169530 & 3.239359 & -1.498234 \\
\hline $\mathrm{H}$ & 1.414605 & 1.971223 & -2.496844 \\
\hline $\mathrm{H}$ & -0.061046 & 3.942363 & -2.010676 \\
\hline $\mathrm{H}$ & 0.117482 & 2.910635 & 0.857263 \\
\hline $\mathrm{H}$ & 1.383314 & 4.605862 & 0.624458 \\
\hline $\mathrm{H}$ & -2.149639 & 3.722887 & 1.139298 \\
\hline $\mathrm{H}$ & -2.030061 & 4.674064 & -0.359338 \\
\hline $\mathrm{H}$ & -2.976342 & 2.766981 & -1.367826 \\
\hline $\mathrm{H}$ & -0.659254 & 1.102456 & 0.910409 \\
\hline $\mathrm{H}$ & -0.957230 & -2.123396 & 2.315783 \\
\hline $\mathrm{H}$ & -0.029882 & -0.598735 & 2.451592 \\
\hline $\mathrm{H}$ & -1.336089 & -0.978893 & 3.599390 \\
\hline $\mathrm{H}$ & -5.116668 & -0.549787 & 0.541818 \\
\hline $\begin{array}{l}6 \\
d\end{array}$ & 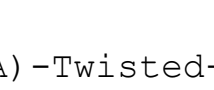 & & \\
\hline $\mathrm{C}$ & -3.666913 & 0.550091 & 1.627871 \\
\hline $\mathrm{C}$ & -2.356090 & 0.928870 & 1.279289 \\
\hline $\mathrm{C}$ & -2.242403 & 2.003782 & 0.397218 \\
\hline $\mathrm{N}$ & -3.250081 & 2.732743 & -0.103880 \\
\hline $\mathrm{C}$ & -4.429397 & 2.305745 & 0.338845 \\
\hline $\mathrm{N}$ & -4.692176 & 1.277579 & 1.160747 \\
\hline $\mathrm{N}$ & -0.898937 & 2.128292 & 0.157396 \\
\hline $\mathrm{C}$ & -0.283047 & 1.146436 & 0.890655 \\
\hline $\mathrm{N}$ & -1.115887 & 0.402831 & 1.580640 \\
\hline $\mathrm{C}$ & -0.261498 & 3.022244 & -0.809834 \\
\hline O & 0.809804 & 2.318923 & -1.402821 \\
\hline $\mathrm{C}$ & 2.058071 & 2.965142 & -1.12637 \\
\hline
\end{tabular}




\begin{tabular}{|c|c|c|c|}
\hline C & 1.780714 & 3.851083 & 0.095098 \\
\hline & 0.340270 & 4.273734 & -0.168153 \\
\hline & 3.148641 & 1.928469 & -0.974205 \\
\hline & 2.966843 & 1.198031 & 0.235313 \\
\hline & 4.132754 & 0.105917 & 0.650657 \\
\hline & 3.913449 & -0.214731 & 2.098659 \\
\hline & 2.622970 & 4.982681 & 0.167788 \\
\hline & -3.921100 & -0.510114 & 2.438678 \\
\hline & 3.701442 & -1.189104 & -0.270513 \\
\hline & 2.935467 & -2.256159 & 0.290803 \\
\hline & 2.599715 & -3.212138 & -0.859264 \\
\hline & 1.363410 & -2.733790 & -1.408025 \\
\hline & 0.767171 & -1.768985 & -0.552387 \\
\hline & 1.557215 & -1.804900 & 0.754607 \\
\hline & 2.468731 & -4.666669 & -0.449594 \\
\hline & 1.546382 & -4.766036 & 0.627273 \\
\hline & -0.644166 & -2.062386 & -0.389014 \\
\hline & -1.223069 & -3.015642 & 0.415689 \\
\hline & -2.533611 & -2.979823 & 0.415277 \\
\hline & -2.839158 & -1.941777 & -0.444322 \\
\hline & -1.681250 & -1.362917 & -0.957430 \\
\hline & -1.619652 & -0.327553 & -1.805290 \\
\hline & -2.831509 & 0.119896 & -2.121878 \\
\hline & -4.025655 & -0.331330 & -1.707469 \\
\hline & -4.059088 & -1.368389 & -0.854833 \\
\hline & -2.865816 & 0.964460 & -2.806522 \\
\hline & 5.448414 & 0.586887 & 0.114435 \\
\hline & 1.441986 & -5.698551 & 0.865394 \\
\hline & -5.250377 & -1.799009 & -0.384586 \\
\hline & 3.457584 & -5.040040 & -0.152597 \\
\hline & 2.119139 & -5.244429 & -1.313675 \\
\hline & 3.365677 & -3.140623 & -1.638111 \\
\hline H & 3.506856 & -2.743313 & 1.086055 \\
\hline $\mathrm{H}$ & 1.148837 & -2.555133 & 1.433630 \\
\hline 1. & 1.580045 & -0.845308 & 1.267518 \\
\hline & 0.809999 & -0.784342 & -1.032632 \\
\hline J & -0.613580 & -3.727257 & 0.957831 \\
\hline 4 & -5.299643 & 2.853776 & -0.013776 \\
\hline $\mathrm{H}$ & 4.121663 & 2.431788 & -0.959907 \\
\hline $\mathrm{H}$ & 3.125654 & 1.247569 & -1.834914 \\
\hline $\mathrm{I}$ & 2.323485 & 3.616200 & -1.972098 \\
\hline $\mathrm{H}$ & 1.855035 & 3.255173 & 1.013029 \\
\hline $\mathrm{H}$ & 3.456999 & 4.733844 & 0.593252 \\
\hline $\mathrm{H}$ & -0.205502 & 4.587659 & 0.723373 \\
\hline $\mathrm{H}$ & 0.343596 & 5.093913 & -0.894269 \\
\hline $\mathrm{H}$ & -1.027474 & 3.248569 & -1.556051 \\
\hline $\mathrm{H}$ & 0.794731 & 1.032738 & 0.851608 \\
\hline $\mathrm{H}$ & -4.862758 & -0.881840 & 2.388334 \\
\hline $\mathrm{H}$ & -3.191439 & -1.210761 & 2.512168 \\
\hline $\mathrm{H}$ & -6.092490 & -1.460181 & -0.830590 \\
\hline $\mathrm{H}$ & -5.299572 & -2.691666 & 0.087916 \\
\hline 6 & & & \\
\hline \multicolumn{4}{|c|}{$d($ ApC $)-$ Twisted-pcm } \\
\hline $\mathrm{C}$ & -0.675478 & -1.604180 & -0.747472 \\
\hline N & -1.039429 & -2.673287 & 0.013888 \\
\hline $\mathrm{C}$ & -2.388196 & -3.087471 & 0.073941 \\
\hline & -3.317614 & -2.347234 & -0.591240 \\
\hline
\end{tabular}




\begin{tabular}{|c|c|c|c|}
\hline C & -2.953103 & -1.301811 & -1.319698 \\
\hline C & -1.586034 & -0.902373 & -1.464252 \\
\hline C & -0.030745 & -3.397203 & 0.808352 \\
\hline 0 & 0.690708 & -2.448030 & 1.57842 \\
\hline C & 2.092762 & -2.574934 & 1.33641 \\
\hline C & 2.179841 & -3.184040 & -0.06556 \\
\hline C & 1.011970 & -4.162578 & -0.02936 \\
\hline C & 2.760722 & -1.226931 & 1.50704 \\
\hline O & 2.301877 & -0.310088 & 0.52334 \\
\hline $\mathrm{P}$ & 3.051433 & -0.149872 & -0.93092 \\
\hline 0 & 1.982992 & -0.280101 & -1.97934 \\
\hline O & 3.431403 & -3.766690 & -0.34107 \\
\hline O & -2.683652 & -4.095997 & 0.72257 \\
\hline $\mathrm{N}$ & -3.923296 & -0.615937 & -1.96384 \\
\hline O & 3.567183 & 1.397641 & -0.84849 \\
\hline C & 2.731049 & 2.532891 & -0.59173 \\
\hline $\mathrm{C}$ & 2.413795 & 2.692703 & 0.91777 \\
\hline O & 0.992402 & 2.544709 & 1.05928 \\
\hline $\mathrm{C}$ & 0.474056 & 1.959923 & -0.12711 \\
\hline C & 1.341432 & 2.528294 & -1.24235 \\
\hline $\mathrm{N}$ & -0.941552 & 2.246915 & -0.22050 \\
\hline C & -1.901421 & 1.577731 & 0.50511 \\
\hline $\mathrm{C}$ & -3.118354 & 2.010189 & -0.00857 \\
\hline $\mathrm{N}$ & -2.928952 & 2.915761 & -1.03471 \\
\hline $\mathrm{C}$ & -1.626532 & 3.024289 & -1.12660 \\
\hline $\mathrm{N}$ & -1.720594 & 0.666367 & 1.47207 \\
\hline $\mathrm{C}$ & -2.878860 & 0.181248 & 1.90642 \\
\hline $\mathrm{N}$ & -4.122600 & 0.497019 & 1.50669 \\
\hline $\mathrm{C}$ & -4.273692 & 1.411282 & 0.53721 \\
\hline $\mathrm{H}$ & -2.819862 & -0.571507 & 2.68924 \\
\hline $\mathrm{C}$ & 2.766370 & 4.064492 & 1.46594 \\
\hline O & 2.106660 & 5.088622 & 0.73976 \\
\hline O & 4.270250 & -1.033304 & -0.95726 \\
\hline $\mathrm{H}$ & 1.152456 & 4.929508 & 0.82 \\
\hline $\mathrm{N}$ & -5.512100 & 1.687844 & 0.0761 \\
\hline $\mathrm{H}$ & 3.840509 & 4.240126 & 1.362 \\
\hline $\mathrm{H}$ & 2.505696 & 4.112334 & 2.530 \\
\hline $\mathrm{H}$ & 2.928075 & 1.910112 & 1.4 \\
\hline $\mathrm{H}$ & 3.318107 & 3.380099 & -0.9503 \\
\hline $\mathrm{H}$ & 1.069499 & 3.572942 & -1.4 \\
\hline $\mathrm{H}$ & 1.294090 & 1.951409 & -2.16 \\
\hline $\mathrm{H}$ & 0.557649 & 0.872595 & -0.0 \\
\hline $\mathrm{H}$ & -1.109974 & 3.646408 & -1.8445 \\
\hline $\mathrm{H}$ & 3.846814 & -1.351960 & 1.44 \\
\hline $\mathrm{H}$ & 2.503981 & -0.803612 & 2.4824 \\
\hline $\mathrm{H}$ & 2.535907 & -3.281813 & 2.05 \\
\hline $\mathrm{H}$ & 1.971001 & -2.421124 & -0.81987 \\
\hline $\mathrm{H}$ & 4.002995 & -3.020711 & -0.61 \\
\hline $\mathrm{H}$ & 0.629306 & -4.437185 & -1.014 \\
\hline $\mathrm{H}$ & 1.310758 & -5.072470 & 0.502 \\
\hline $\mathrm{H}$ & -0.602305 & -4.052767 & 1.46174 \\
\hline $\mathrm{H}$ & 0.372000 & -1.321004 & -0.7464 \\
\hline $\mathrm{H}$ & -1.288424 & -0.054944 & -2.0719 \\
\hline $\mathrm{H}$ & -4.883937 & -0.788096 & -1.69438 \\
\hline $\mathrm{H}$ & -3.719372 & 0.296460 & -2.35107 \\
\hline $\mathrm{H}$ & -6.307987 & 1.351634 & 0.6022 \\
\hline $\mathrm{H}$ & -5.651591 & 2.496273 & -0 \\
\hline
\end{tabular}


64

d (ApG) -Twisted-pcm

C $\quad-3.614434 \quad-1$.

$-1.961752 \quad-0.804883$

$-2.318183 \quad-2.355768$

$-0.421347$

$-1.266245$

$-1.608612$

$-1.371080$

$-0.593095$

$-2.638103$

$-3.747446$

$-0.342912$

$-0.947170$

$-1.815190$

$-2.128584$

$-0.129651$

$-0.950148$

$-1.675826$

$-0.550877$

$-2.137028$

$-0.383681$

0.419957

0.428774

$-1.850396$

1.218323

1.944327

3.236944

3.445893

2.021477

4.067789

4.273677

5.495110

3.310283

2.418805

$-2.807352$

2.944998

2.991829

1.726272

$-3.171981$

$-3.338061$

$-1.623085$

$-0.551258$

$-2.447611$

$-1.452285$

$-2.775543$

$-0.923356$

0.267291

0.748314

$-1.590786$

$-0.653430$

$-0.244374$

0.669664

0.713753

1.372352

0.200298

$-4.250206$

$-0.576464$

0.497065

0.466864

$-2.834609$

1.560692

0.309141

2.249846

$-0.936368$

3.049539

$-1.138446$

2.161188

2.715349

$-1.344167$

$-0.565226$

$-0.199470$

1.289183

4.096577

3.951195

$-0.819982$

$-0.270140$

0.021754

1.804488

0.209488

1.486778

0.440117

$-2.371748$

0.490303

1.409643

$-1.042236$

$-0.313877$

0.197527

1.770275

0.999645

1.033410

$-3.445672$

2.044112

$-0.169899$

1.511339

0.216069

0.511771

$-4.664491$

$-3.581316$

$-3.788887$

$-5.748882$

1.921193

4.102160

2.438479

$-4.725373$

4.343399

3.036625

3.981466

4.077885

1.730899

1.915177

1.100251

0.162905

3.852391

3.102803

1.861228

$-0.096824$

1.153135

1.819423

$-1.006466$

2.618063

1.912568

5.214252

0.285483

$-0.357688$

0.018797

2.152478

0.691655

$-2.589812$

$-0.346541$

$-4.496308$

$-0.297632$

$-4.833899$

$-1.463657$

$-2.565739$

$-2.273673$

$-1.698020$

1.043668

1.405335

$-2.411585$

1.292470

$-0.654427$

$-0.989806$

0.952955

$-3.786301$

2.928376

$-0.953362$

1.532950

$-1.760481$

3. 678284

$-2.031144$

0.976841

1.455569
2.790840

3.437253

0.439035 


\begin{tabular}{|c|c|c|c|}
\hline $\mathrm{H}$ & -0.803177 & 4.371831 & 0.596499 \\
\hline $\mathrm{H}$ & -0.329942 & 4.851897 & -1.052820 \\
\hline $\mathrm{H}$ & -1.340910 & 2.751305 & -1.589185 \\
\hline $\mathrm{H}$ & 0.768125 & 1.060321 & 1.018827 \\
\hline $\mathrm{H}$ & -5.575296 & 0.124543 & 1.383904 \\
\hline $\mathrm{H}$ & -5.685900 & 2.776965 & -0.881340 \\
\hline $\mathrm{H}$ & -6.611594 & 1.798933 & 0.159646 \\
\hline $\mathrm{H}$ & -5.612244 & -2.124372 & -0.493490 \\
\hline $\begin{array}{c}\mathrm{H} \\
63\end{array}$ & -4.643182 & -3.137786 & 0.500483 \\
\hline \multicolumn{4}{|c|}{$d($ ApT $)-T w i s t e d-p c m$} \\
\hline $\mathrm{C}$ & 4.241606 & 1.516436 & -0.562104 \\
\hline $\mathrm{C}$ & 3.061001 & 2.111878 & -0.067729 \\
\hline $\mathrm{C}$ & 1.863413 & 1.566149 & -0.516558 \\
\hline $\mathrm{N}$ & 1.723531 & 0.558201 & -1.392310 \\
\hline $\mathrm{C}$ & 2.901656 & 0.095331 & -1.794364 \\
\hline $\mathrm{N}$ & 4.130601 & 0.502090 & -1.432852 \\
\hline $\mathrm{N}$ & 2.831358 & 3.137097 & 0.828093 \\
\hline $\mathrm{C}$ & 1.524825 & 3.198058 & 0.912979 \\
\hline $\mathrm{N}$ & 0.875499 & 2.274641 & 0.127656 \\
\hline $\mathrm{C}$ & -0.530808 & 1.920391 & 0.069554 \\
\hline O & -1.064632 & 2.348031 & -1.171577 \\
\hline C & -2.462208 & 2.629181 & -1.032058 \\
\hline C & -2.795271 & 2.535205 & 0.476650 \\
\hline C & -1.409817 & 2.573614 & 1.129501 \\
\hline O & -3.623545 & 1.408227 & 0.788434 \\
\hline $\mathrm{P}$ & -3.103525 & -0.135233 & 0.897167 \\
\hline O & -4.322421 & -1.019844 & 0.934478 \\
\hline C & -2.740798 & 4.002487 & -1.608850 \\
\hline O & -2.100970 & 4.967445 & -0.785927 \\
\hline $\mathrm{H}$ & 2.876109 & -0.726388 & -2.507459 \\
\hline O & -2.350513 & -0.311970 & -0.550654 \\
\hline C & -2.810283 & -1.235253 & -1.526337 \\
\hline $\mathrm{C}$ & -2.151616 & -2.586050 & -1.336978 \\
\hline O & -0.750322 & -2.470578 & -1.585298 \\
\hline C & -0.035421 & -3.428652 & -0.821864 \\
\hline $\mathrm{C}$ & -1.067316 & -4.137457 & 0.077468 \\
\hline C & -2.237434 & -3.161704 & 0.078767 \\
\hline $\mathrm{N}$ & 1.036069 & -2.730790 & -0.095478 \\
\hline C & 0.733563 & -1.739729 & 0.822182 \\
\hline C & 1.660013 & -1.047943 & 1.519570 \\
\hline C & 3.064074 & -1.332511 & 1.259261 \\
\hline $\mathrm{N}$ & 3.284587 & -2.322567 & 0.306235 \\
\hline C & 2.347440 & -3.035182 & -0.411086 \\
\hline O & 2.679263 & -3.868163 & -1.245273 \\
\hline O & 4.014652 & -0.776426 & 1.798926 \\
\hline $\mathrm{C}$ & 1.316592 & 0.012821 & 2.521596 \\
\hline O & -3.486620 & -3.741839 & 0.368901 \\
\hline O & -2.042552 & -0.249667 & 1.955912 \\
\hline $\mathrm{H}$ & -2.297967 & 5.852106 & -1.125110 \\
\hline $\mathrm{N}$ & 5.469529 & 1.956838 & -0.212916 \\
\hline $\mathrm{H}$ & -3.826878 & 4.162554 & -1.629476 \\
\hline $\mathrm{H}$ & -2.358003 & 4.047388 & -2.635848 \\
\hline $\mathrm{H}$ & -3.039320 & 1.879739 & -1.584139 \\
\hline $\mathrm{H}$ & -3.390635 & 3.393920 & 0.790048 \\
\hline $\mathrm{H}$ & -1.143892 & 3.629632 & 1.230813 \\
\hline $\mathrm{H}$ & -1.367767 & 2.069388 & 2.094683 \\
\hline
\end{tabular}




\begin{tabular}{|c|c|c|c|}
\hline & -0.563817 & 0.828815 & 0.129725 \\
\hline & 0.982839 & 3.885969 & 1.546851 \\
\hline & -3.897514 & -1.353386 & -1.469801 \\
\hline & -2.545412 & -0.824607 & -2.505167 \\
\hline & -2.601702 & -3.303515 & -2.040583 \\
\hline & -2.033228 & -2.376245 & 0.809557 \\
\hline & -4.058158 & -2.990279 & 0.632827 \\
\hline & -0.670501 & -4.355760 & 1.071352 \\
\hline & -1.372591 & -5.077339 & -0.394463 \\
\hline & 0.478676 & -4.122877 & -1.484245 \\
\hline & -0.322266 & -1.514725 & 0.950867 \\
\hline & 1.87938 & 0.930839 & 2.318849 \\
\hline & 0.243492 & 0.224559 & 2.493333 \\
\hline & 1.586166 & -0.307923 & 3.534410 \\
\hline & 4.252803 & -2.524781 & 0.07287 \\
\hline & 6.26641 & 1.377440 & -0.442516 \\
\hline & 5.560442 & 2.571247 & 0.585149 \\
\hline & & & \\
\hline & In & $-\mathrm{pcm}$ & \\
\hline & -0.24656400 & -0.47459200 & -3.13057700 \\
\hline & 0.13537700 & -0.35179100 & -2.24941700 \\
\hline & 1.47355200 & -0.82370500 & -2.25215300 \\
\hline & 2.09332300 & -0.25734100 & -2.96211000 \\
\hline & 1.52435400 & -1.88688100 & -2.51553000 \\
\hline & 1.99824100 & -0.63079900 & -0.83981700 \\
\hline & 1.31900600 & -1.11130900 & -0.12613200 \\
\hline & 3.29148100 & -1.23926600 & -0.74664600 \\
\hline & 4.23407600 & -0.29359100 & -0.26056100 \\
\hline & 4.34583800 & -0.38 & 3800 \\
\hline & 5.52682300 & -0.61362000 & -0.82 \\
\hline & 5.86271900 & -0.68708200 & -2.16314300 \\
\hline & 5.13364700 & -0.46624500 & -2.93243800 \\
\hline & 7.09507500 & -1.05279300 & -2.38176200 \\
\hline & 7.62 & -1.24176800 & -1.11270900 \\
\hline & 8.86870500 & -1.63471000 & -0.62 \\
\hline & 9.70046400 & -1.86984600 & -1.29040900 \\
\hline & 9.08148600 & -1.73886800 & 0.67910400 \\
\hline & 8.05791400 & -1.45449100 & 1.52094400 \\
\hline & 8.343460 & -1.52463800 & 2.85867500 \\
\hline & 9.14861 & 59000 & 89000 \\
\hline & 7.55010500 & -1.60389300 & 3.48154300 \\
\hline & 6.81996400 & -1.07003900 & 1.18151900 \\
\hline & 6.65382900 & -0.97894600 & -0.13226900 \\
\hline & 3.67470100 & 1.06811100 & -0.66035600 \\
\hline & 3.89879000 & 1.27174200 & -1.71307700 \\
\hline & 4.05295500 & 1.88786600 & -0.04779700 \\
\hline & 2.17826700 & 0.84842400 & -0.46690700 \\
\hline & 1.56225800 & 1.49127400 & -1.10369700 \\
\hline & 1.81468000 & 1.01249900 & 0.90130400 \\
\hline & 1.13362000 & 2.44176100 & 1.35329500 \\
\hline & 0.70219800 & 2.24875700 & 2.77607000 \\
\hline & 1.99559000 & 3.59023400 & 0.92143300 \\
\hline & -0.17223400 & 2.47571300 & 0.35472900 \\
\hline & -1.11926100 & 1.42140500 & 0.44452500 \\
\hline & 550400 & 1.43033800 & 1.42971300 \\
\hline & -0.63258700 & 0.44904500 & 0.29909400 \\
\hline & -2.13721300 & 1.62836800 & -0.6648180 \\
\hline
\end{tabular}


$\mathrm{H} \quad-1.61993900$

O -3.01554400

C -4.34542600

$\mathrm{H} \quad-4.93742700$

N -4.96543000

C -4.49964700

$\mathrm{N}-5.26602800$

C -6.29263300

C -6.11912700

C -4.22411200

$\mathrm{H}-3.99772000$

$\mathrm{H}-5.13281700$

C -3.02819200

$\mathrm{H} \quad-2.51199300$

O -3.39605000

$\mathrm{H} \quad-3.96765000$

$\mathrm{H} \quad-3.57816000$

C -7.43562200

$\mathrm{N}-8.21692800$

C -7.95261800

$\mathrm{N}-6.90739700$

O -7.77987300

$\mathrm{H} \quad-9.03342500$

$\mathrm{N} \quad-8.80414400$

$\mathrm{H} \quad-9.75362300$

$\mathrm{H} \quad-8.70642000$

63

d (2pT) -Unstacked-pcm

$\mathrm{H} \quad 0.61080600$

O 0.26945100

C -1.09254500

$\mathrm{H} \quad-1.70615100$

$\mathrm{H}-1.21349800$

C -1.54839400

$\mathrm{H} \quad-0.87161900$

O -2.87188700

C $\quad-3.72751700$

$\mathrm{H} \quad-3.78668400$

$\mathrm{N} \quad-5.06491000$

C $\quad-5.47058100$

$\mathrm{H} \quad-4.76604500$

$\mathrm{N}-6.73388500$

C -7.21155300

C -8.45370300

$\mathrm{H}-9.33093400$

$\mathrm{N} \quad-8.60811400$

C $\quad-7.52736300$

$\mathrm{N} \quad-7.75039500$

$\mathrm{H} \quad-8.57752900$

$\mathrm{H} \quad-6.93418500$

$\mathrm{N}-6.28475400$

C -6.17810000

C -3.10811500

$\mathrm{H}-3.37524600$

$\mathrm{H} \quad-3.40201900$

C -1.61963800

$\mathrm{H}-1.00049400$
1.69195000

0.49304900

0.89240700

0.94711500

$-0.13486200$

$-0.64799500$

$-1.58659000$

$-1.70192800$

$-0.81302400$

2. 25387000

2.12765800

2.85265600

2.85299800

3.62870000

3.33364900

4.10935000

$-0.29365200$

$-2.56417000$

$-2.33396000$

$-1.41658600$

$-0.62187200$

$-3.41556100$

$-2.93304400$

$-1.39135300$

$-1.72054000$

$-0.58836900$

$-1.15758400$

$-0.81951500$

$-0.45544800$

$-1.32385700$

0.31906100

0.08943700

0.89012300

0.61647500

0.04076800

0.68136900

$-0.01124600$

$-0.64751200$

$-1.20828100$

$-0.48571600$

0.31134400

0.85625700

0.69163900

1.61138500

1.83342500

2. 57258800

3.15484000

2.98611500

1.36898400

0.62090400

$-1.31595800$

$-2.04240000$

$-1.70713900$

$-0.99110200$

$-1.85469900$
$-1.62741500$

$-0.68610700$

$-0.39493700$

$-1.31332100$

0.41892400

1. 61188000

2.10029200

1.18537500

0.13250900

0.28576900

1. 35018900

0.19206800

$-0.45077700$

0.12422300

$-1.73707500$

$-1.63054300$

2.05485400

1.17107100

0.01999100

$-0.96661000$

$-0.95075600$

1.98490900

$-0.06545400$

$-2.02574700$

$-1.90655600$

$-2.63452600$

$-3.16449300$

$-2.32388500$

$-2.48573600$

$-2.76523700$

$-3.25243700$

$-1.14334800$

$-0.82316200$

$-1.28920300$

$-0.31172100$

0.57478800

$-0.85832800$

$-2.01710800$

$-2.61828000$

$-2.29669800$

$-1.26554100$

$-0.95708500$

$-1.57892100$

0.12721700

0.91460800

2. 04593000

2.03841800

2.47772700

0.72528900

$-0.36603600$

0.00895400

$-0.76622100$

0.98410800

$-0.05357000$

$-0.31623900$ 


\begin{tabular}{|c|c|c|c|}
\hline & -1.17354300 & -0.41825500 & 1.17288600 \\
\hline & -0.39466300 & -1.39552700 & 2.24452100 \\
\hline & 0.10431100 & -0.48707400 & 3.32777300 \\
\hline & -1.21693600 & -2.61638700 & 2.53027900 \\
\hline & 0.85513700 & -1.91838100 & 1.31195000 \\
\hline & 1.75347900 & -0.95361700 & 0.78515600 \\
\hline & 2.32611300 & -0.48883200 & 160007500 \\
\hline & 1.21006800 & -0.17010000 & 0.24209100 \\
\hline & 2.67168400 & -1.67341300 & -0.18898400 \\
\hline & 2.06698900 & -2.19721600 & -0.93554900 \\
\hline & 3.48068900 & -0.69275500 & -0.85784200 \\
\hline & 4.85184300 & -0.91310500 & -0.57812000 \\
\hline & 34300 & 1675900 & \\
\hline & 5.50792100 & 0.38952600 & -0.45156400 \\
\hline & 4.93596300 & 5817400 & 3717300 \\
\hline & 3.99050300 & 1.08350500 & 0.78721300 \\
\hline & 5.47053600 & 2.59194900 & 0.52586000 \\
\hline & 4.84441300 & 63600 & 6200 \\
\hline & 4.6 & 300 & 0.782 \\
\hline & 3.91119400 & 3.29787800 & 1.81647800 \\
\hline & 5.52047500 & 3.95439600 & 2.18297100 \\
\hline & 6.72875200 & 2.89350400 & -0.14716800 \\
\hline & 7.33067800 & 3.95787300 & -0.07215500 \\
\hline & 725300 & 11500 & -0 . \\
\hline & 8.11621200 & 871300 & 6400 \\
\hline & 6.70168400 & 0.59886200 & -1.11382900 \\
\hline & 7.25465000 & -0.24318700 & -1.80759500 \\
\hline & 4.87859900 & -1.76009100 & 0.69174100 \\
\hline & 4.7 & -1 & 00 \\
\hline & 5.80412 & -2.33092500 & 63600 \\
\hline & 3.64529500 & -2.63833900 & 0.49058300 \\
\hline & 3.23424700 & -3.02761500 & 1.42778200 \\
\hline & 3.9000110 & -3.69238800 & -0.42851400 \\
\hline & 4.51521600 & -4 & -0 . \\
\hline & & & \\
\hline \multicolumn{4}{|c|}{$d(A p G)$-Unstacked-pcm } \\
\hline & -0.24110600 & -1.03365800 & -2.80115000 \\
\hline & 0.11872100 & -0.73947400 & -1.95179200 \\
\hline & 1.45476800 & -1.20065200 & -1.82669400 \\
\hline & 2.093 & 00 & -2.62070400 \\
\hline & 1.508 & -2.2 & $-1 . \varepsilon$ \\
\hline & 1.94505700 & -0.72650500 & -0.46948600 \\
\hline & 1.24776400 & -1.05688700 & 0.30906400 \\
\hline & 3.23450500 & -1.29990700 & -0.22523200 \\
\hline & 4.165741 & -0.27455400 & 0.08929200 \\
\hline & 4.25254 & -0.15147100 & 1.17406200 \\
\hline & 5.471891 & -0.69514800 & -0.37035300 \\
\hline & 5.833988 & -1.04133600 & -1.65206300 \\
\hline & 5.12178700 & -1.00469200 & -2.46646600 \\
\hline & 7.08150100 & -1.42235400 & -1.76026000 \\
\hline & 7.57415200 & -1.32519100 & -0.47342000 \\
\hline & 8.84228400 & -1.58310900 & 0.08768100 \\
\hline & 9.87557500 & -2.03866300 & -0.64730200 \\
\hline & 9.00971700 & -1.39084100 & 1.40680000 \\
\hline & 7.96479200 & -0.96321600 & 2.13232300 \\
\hline & 8.16051400 & -0.82779800 & 3.19308600 \\
\hline & 6.73041400 & -0.68300500 & 1.7248830 \\
\hline
\end{tabular}




C $\quad 3.61959100$

$\mathrm{H} \quad 3.87627100$

$\mathrm{H} \quad 3.97974800$

C 2.11748900

$\mathrm{H} \quad 1.52171000$

O 1.71367800

P $\quad 1.02719900$

O 0.55415000

O 1.90663400

O -0.25008000

C -1.21039600

$\mathrm{H}-1.76270400$

$\mathrm{H} \quad-0.72470600$

C -2.14506500

$\mathrm{H} \quad-1.55767400$

O -3.02941500

C -4.37327500

$\mathrm{H}-4.90046400$

$\mathrm{N} \quad-5.05553600$

C -4.70568500

$\mathrm{N} \quad-5.48773000$

C -6.40213600

C -6.14482600

C -4.28836400

$\mathrm{H}-4.13781800$

$\mathrm{H} \quad-5.18360500$

C -3.03893500

$\mathrm{H} \quad-2.55809100$

O -3.31201300

$\mathrm{H}-3.88374300$

$\mathrm{H} \quad-3.85215200$

C -7.50253300

$\mathrm{N} \quad-8.15827700$

C $\quad-7.82026200$

$\mathrm{N}-6.81363900$

O -7.90172900

$\mathrm{H}-8.93387100$

$\mathrm{N}-8.54940500$

$\mathrm{H} \quad-9.49322300$

$\mathrm{H} \quad-8.41411200$

H 10.79438000

$\mathrm{H} \quad 9.81015600$

63

$$
\text { d (ApT) -Unstacked-pcm }
$$

$\begin{array}{rr}\mathrm{H} & 0.52958300 \\ \mathrm{O} & 0.22856500 \\ \mathrm{C} & -1.14016600 \\ \mathrm{H} & -1.76370000 \\ \mathrm{H} & -1.29845500 \\ \mathrm{C} & -1.53621200 \\ \mathrm{H} & -0.84412900 \\ \mathrm{O} & -2.86326100 \\ \mathrm{C} & -3.68408000 \\ \mathrm{H} & -3.71841400 \\ \mathrm{~N} & -5.03976100 \\ \mathrm{C} & -5.48394500\end{array}$

$$
-0.24042900
$$

$-0.10590500$

0.26758300

$-0.51800500$

1.19997400

0.47325700

1.17956700

1.01036600

0.20254000

0.60714300

0.26845400

$-0.07801100$
0.40665800

$-0.58235000$

$-1.64728700$

$-0.12803500$

$-0.39362500$

$-1.16316400$

0.90333800

1.04365600

2. 46338100

0.41709500

0.02179000

0.29676600

1. 21510200

0.42536400

$-0.89942200$

$-1.80770700$

$-0.71058600$

$-0.61378000$

$-1.55636900$

0.37943500

1.70399300

2. 34194000

1. 39240300

0.16718200

$-0.27709700$

0.79880600

$-0.57654600$

$-1.05193000$

$-0.64861400$

$-2.43911400$

$-2.56607200$

2.12877500

1. 49011400

0.24784100

$-0.91214600$

$-1.00052100$

2.45908600

0.24004900

$-2.01934400$

$-1.91266900$

$-2.80368900$

$-0.22815600$

$-1.65559200$

$-3.23722100$

$-2.32691600$

$-2.33782100$

$-2.78819400$

$-2.89276100$

$-0.88600100$

$-0.41300900$

$-0.84598700$

$-0.01417000$

1.00290900

$-0.51544100$

$-1.77110600$ 
$\mathrm{H} \quad-4.80654100$

$\mathrm{N} \quad-6.76282600$

C -7.19063000

C -8.45016000

$\mathrm{N} \quad-9.55964500$

$\mathrm{N} \quad-8.54329800$

C $\quad-7.43661000$

$\mathrm{H} \quad-7.57402700$

$\mathrm{N}-6.20237300$

C -6.13648400

C -3.04936700

$\mathrm{H}-3.34020900$

H -3.30694100

C -1.56503900

$\mathrm{H}-0.95196800$

O -1.07757300

P -0.29732300

O 0.25926800

O -1.14645900

O $\quad 0.90880600$

C $\quad 1.81973100$

$\mathrm{H} \quad 2.44853500$

$\mathrm{H} \quad 1.28398000$

C $\quad 2.66057300$

$\mathrm{H} \quad 2.00005000$

O 3.47511200

C $\quad 4.84811300$

$\mathrm{H} \quad 5.26447800$

$\mathrm{N} \quad 5.56790500$

C $\quad 5.08381600$

$\mathrm{H} \quad 4.15311700$

C $\quad 5.68065500$

C $\quad 5.14791700$

$\mathrm{H} \quad 4.94091700$

$\mathrm{H} \quad 4.22522300$

$\mathrm{H} \quad 5.87859800$

C $\quad 6.91180600$

O 7.56300800

$\mathrm{N} \quad 7.33122100$

$\mathrm{H} \quad 8.18984400$

C $\quad 6.73137800$

O 7.20810300

C $\quad 4.90600600$

$\mathrm{H} \quad 4.86307600$

$\mathrm{H} \quad 5.80927500$

C $\quad 3.62597300$

$\mathrm{H} \quad 3.24876800$

O 3.78486400

$\mathrm{H} \quad 4.39640500$

$\mathrm{H}-10.40629300$

$\mathrm{H} \quad-9.48301300$ 30

2|A-Dimer-Twisted-pcm

$\begin{array}{rrr}\mathrm{N} & 0.003965 & -0.005533 \\ \mathrm{C} & -0.018669 & 0.023453 \\ \mathrm{~N} & 1.036198 & 0.020270 \\ \mathrm{C} & 2.193561 & -0.050707\end{array}$

1.46722600

1.46722600
$-2.51827700$

$-1.95228100$

$-0.74613800$

$-0.28525200$

$-1.04684700$

0.96708500

1.72536000

2.72803600

1.41180900

0.15742300

$-0.05249300$

$-0.97148700$

0.80821600

$-0.08418300$

$-0.56561300$

1.22737400

2.02987000

3.26832000

2.06908000

0.96221000

0.62975300

1. 49842400

0.31944400

$-0.53660800$

$-1.35073000$

$-0.99830300$

$-0.86768400$

$-1.82854400$

$-0.48103100$

0.54862300

0.97986800

0.98178000

2.09381500

1.74007700

2.49659100

2.90480600

0.31854700

0.60010100

$-0.70718000$

$-1.18717000$

$-1.15109100$

$-2.05555400$

0.16255100

1.17783600

0.07123700

$-0.16152100$

0.68265300

$-1.31521300$

$-1.10766000$

$-0.72332100$

$-2.03055300$

0.000929

1.355435

2.180052

1.532093 


\begin{tabular}{|c|c|c|c|}
\hline $\mathrm{C}$ & 2.359384 & -0.120084 & 0.137777 \\
\hline C & 1.184436 & -0.078857 & -0.607033 \\
\hline $\mathrm{N}$ & 3.459001 & -0.108535 & 2.042828 \\
\hline C & 4.311076 & -0.237683 & 0.971298 \\
\hline $\mathrm{N}$ & 3.703356 & -0.245425 & -0.185805 \\
\hline $\mathrm{N}$ & -1.262811 & 0.106533 & 1.932968 \\
\hline $\mathrm{H}$ & -2.014102 & -0.272081 & 1.368629 \\
\hline $\mathrm{H}$ & -1.300617 & -0.170258 & 2.906454 \\
\hline $\mathrm{H}$ & 3.717513 & -0.116749 & 3.021840 \\
\hline $\mathrm{H}$ & 5.380071 & -0.317973 & 1.115976 \\
\hline $\mathrm{H}$ & 1.184652 & -0.121377 & -1.694752 \\
\hline $\mathrm{N}$ & 0.060459 & -3.174680 & -1.257641 \\
\hline C & -1.092894 & -3.120645 & -0.570945 \\
\hline $\mathrm{N}$ & -1.274265 & -3.081212 & 0.745223 \\
\hline $\mathrm{C}$ & -0.106751 & -3.111688 & 1.404996 \\
\hline C & 1.164108 & -3.172657 & 0.833244 \\
\hline $\mathrm{C}$ & 1.213862 & -3.188637 & -0.574976 \\
\hline $\mathrm{N}$ & 0.121428 & -3.085264 & 2.755178 \\
\hline $\mathrm{C}$ & 1.480561 & -3.126239 & 2.928664 \\
\hline $\mathrm{N}$ & 2.147540 & -3.182032 & 1.802210 \\
\hline $\mathrm{H}$ & -1.994279 & -3.103569 & -1.178482 \\
\hline $\mathrm{H}$ & -0.576948 & -3.039244 & 3.487326 \\
\hline $\mathrm{H}$ & 1.921090 & -3.110555 & 3.916355 \\
\hline $\mathrm{N}$ & 2.386908 & -3.241212 & -1.253778 \\
\hline $\mathrm{H}$ & 3.221208 & -2.931978 & -0.768028 \\
\hline $\mathrm{H}$ & 2.348838 & -3.014522 & -2.240233 \\
\hline \multicolumn{4}{|c|}{28} \\
\hline \multicolumn{4}{|c|}{2 |C-Dimer-Twisted-pcm } \\
\hline $\mathrm{N}$ & -0.329094 & 0.226102 & -0.183107 \\
\hline $\mathrm{C}$ & -0.077440 & 0.104711 & 1.142646 \\
\hline $\mathrm{N}$ & 1.247256 & 0.127564 & 1.505565 \\
\hline $\mathrm{C}$ & -1.593885 & 0.188579 & -0.591554 \\
\hline C & -2.625646 & 0.022177 & 0.327160 \\
\hline $\mathrm{C}$ & -2.223911 & -0.105426 & 1.669076 \\
\hline $\mathrm{N}$ & -0.975171 & -0.074263 & 2.120510 \\
\hline $\mathrm{N}$ & -4.008229 & -0.033174 & 0.225368 \\
\hline $\mathrm{C}$ & -4.412899 & -0.187017 & 1.457327 \\
\hline $\mathrm{N}$ & -3.387093 & -0.238650 & 2.374208 \\
\hline C & -1.261081 & 3.116735 & 2.310836 \\
\hline $\mathrm{N}$ & 0.036032 & 3.087227 & 2.708698 \\
\hline $\mathrm{C}$ & 1.107299 & 3.199815 & 1.816839 \\
\hline $\mathrm{N}$ & 0.816772 & 3.351842 & 0.497495 \\
\hline C & -0.451207 & 3.384206 & 0.098028 \\
\hline C & -1.565053 & 3.269723 & 0.999622 \\
\hline O & 2.257347 & 3.145089 & 2.265265 \\
\hline $\mathrm{N}$ & -0.684507 & 3.538055 & -1.215484 \\
\hline $\mathrm{H}$ & -1.777131 & 0.298354 & -1.658507 \\
\hline $\mathrm{H}$ & -5.444342 & -0.271556 & 1.772088 \\
\hline $\mathrm{H}$ & 1.426696 & 0.357090 & 2.475781 \\
\hline $\mathrm{H}$ & 1.857356 & 0.606697 & 0.853677 \\
\hline $\mathrm{H}$ & -2.008122 & 3.005993 & 3.088689 \\
\hline $\mathrm{H}$ & -2.589696 & 3.291956 & 0.651213 \\
\hline $\mathrm{H}$ & 0.094791 & 3.557240 & -1.859524 \\
\hline $\mathrm{H}$ & -1.620690 & 3.515727 & -1.593396 \\
\hline $\mathrm{H}$ & -3.481341 & -0.353838 & 3.375992 \\
\hline $\mathrm{H}$ & 0.271825 & 2.965423 & 3.687085 \\
\hline
\end{tabular}


2|G-Dimer-Twisted-pcm

\begin{tabular}{|c|c|c|c|}
\hline J & -0.017649 & -0.009426 & 0.048662 \\
\hline 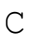 & -0.009276 & -0.003024 & 1.403907 \\
\hline & 1.063984 & -0.019586 & 2.203829 \\
\hline & 2.207107 & -0.053701 & 1.528698 \\
\hline & 2.342839 & -0.068611 & 0.128650 \\
\hline & 1.150681 & -0.042254 & -0.587540 \\
\hline & 3.485326 & -0.091044 & 2.008296 \\
\hline & 4.316581 & -0.134230 & 0.912144 \\
\hline & 3.682431 & -0.118741 & -0.229251 \\
\hline & -1.241023 & 0.069341 & 2.010880 \\
\hline & -0.213707 & -3.059898 & 1.379645 \\
\hline & 1.048540 & -3.208765 & 0.815397 \\
\hline & 1.160753 & -3.226176 & -0.612480 \\
\hline & -0.102991 & -3.059974 & -1.211719 \\
\hline & -1.295818 & -2.895690 & -0.552524 \\
\hline & -1.406527 & -2.908420 & 0.755596 \\
\hline & 2.016823 & -3.318945 & 1.792115 \\
\hline & 1.353330 & -3.237665 & 2.916303 \\
\hline & 0.000338 & -3.087029 & 2.725744 \\
\hline & 2.159164 & -3.357949 & -1.314538 \\
\hline & -2.393536 & -2.664532 & -1.321502 \\
\hline & 3.769219 & -0.110921 & 2.980071 \\
\hline & -0.705289 & -3.001070 & 3.447255 \\
\hline & 1.126603 & -0.058000 & -1.675692 \\
\hline & 5.391201 & -0.172680 & 1.030069 \\
\hline & -1.248472 & -0.209146 & 2.984872 \\
\hline & -1.995485 & -0.334870 & 1.467586 \\
\hline & 1.781574 & -3.279902 & 3.908024 \\
\hline & -0.090290 & -3.024689 & -2.227099 \\
\hline & -3.279470 & -2.756522 & -0.840121 \\
\hline & -2.399071 & -2.981318 & -2.282807 \\
\hline 30 & & & \\
\hline \multicolumn{4}{|c|}{ 2|T-Dimer-Twisted-pcm } \\
\hline $\mathrm{C}$ & -0.006123 & 0.019434 & -0.051574 \\
\hline C & 0.031052 & -0.043585 & 1.296758 \\
\hline $\mathrm{N}$ & 1.209417 & -0.066807 & 2.007500 \\
\hline $\mathrm{C}$ & 2.449205 & -0.040129 & 1.420438 \\
\hline | & 2.403131 & 0.012270 & 0.044016 \\
\hline C & 1.267102 & 0.045245 & -0.766216 \\
\hline 0 & 3.496168 & -0.068050 & 2.053174 \\
\hline 0 & 1.386359 & 0.092787 & -1.984336 \\
\hline N & 2.510903 & -3.148301 & 1.546254 \\
\hline C & 1.304583 & -3.159803 & 0.896007 \\
\hline v & 1.362325 & -3.148998 & -0.458391 \\
\hline c & 0.220633 & -3.212287 & -1.138442 \\
\hline C & -0.995595 & -3.284461 & -0.467178 \\
\hline $\mathrm{C}$ & -0.912736 & -3.271570 & 0.936854 \\
\hline $\mathrm{N}$ & 0.202905 & -3.216840 & 1.656211 \\
\hline $\mathrm{N}$ & -2.209459 & -3.310310 & 1.366420 \\
\hline C & -2.998536 & -3.347796 & 0.238093 \\
\hline $\mathrm{N}$ & -2.319925 & -3.333529 & -0.877098 \\
\hline $\mathrm{H}$ & 0.285539 & -3.194263 & -2.224537 \\
\hline $\mathrm{H}$ & -4.076838 & -3.385409 & 0.315396 \\
\hline $\mathrm{H}$ & 2.499384 & -2.858574 & 2.515893 \\
\hline $\mathrm{H}$ & 3.305170 & -2.825036 & 1.008984 \\
\hline & -0.868514 & -0.078988 & 1.902245 \\
\hline
\end{tabular}




\begin{tabular}{|c|c|c|c|}
\hline $\mathrm{C}$ & -1.272579 & 0.074296 & -0.851143 \\
\hline $\mathrm{H}$ & -2.532047 & -3.320921 & 2.326457 \\
\hline $\mathrm{H}$ & 1.193756 & -0.122574 & 3.019287 \\
\hline $\mathrm{H}$ & 3.302260 & 0.024495 & -0.429631 \\
\hline $\mathrm{H}$ & -2.141417 & -0.089188 & -0.207764 \\
\hline $\mathrm{H}$ & -1.380938 & 1.046597 & -1.343818 \\
\hline $\mathrm{H}$ & -1.272575 & -0.691851 & -1.632405 \\
\hline \multicolumn{4}{|c|}{30} \\
\hline \multicolumn{4}{|c|}{ A|A-Dimer-Twisted-pcm } \\
\hline $\mathrm{N}$ & -0.007236 & 0.063143 & -0.055762 \\
\hline $\mathrm{C}$ & -0.051299 & 0.101980 & 1.285468 \\
\hline $\mathrm{N}$ & 0.958441 & 0.073196 & 2.150589 \\
\hline $\mathrm{C}$ & 1.188869 & -0.029627 & -0.655700 \\
\hline $\mathrm{C}$ & 2.344053 & -0.092663 & 0.148762 \\
\hline $\mathrm{C}$ & 2.140621 & -0.025708 & 1.526798 \\
\hline $\mathrm{N}$ & 3.684138 & -0.251421 & -0.148928 \\
\hline C & 4.267981 & -0.265411 & 1.025391 \\
\hline $\mathrm{N}$ & 3.393769 & -0.120029 & 2.068891 \\
\hline $\mathrm{N}$ & 1.249489 & -0.032799 & -2.008677 \\
\hline $\mathrm{C}$ & -0.089879 & -3.105393 & 1.430981 \\
\hline C & 1.168107 & -3.140810 & 0.829875 \\
\hline C & 1.186174 & -3.133781 & -0.579389 \\
\hline $\mathrm{N}$ & 0.017934 & -3.111934 & -1.236913 \\
\hline C & -1.119629 & -3.079705 & -0.522825 \\
\hline $\mathrm{N}$ & -1.271528 & -3.072216 & 0.797432 \\
\hline $\mathrm{N}$ & 2.172818 & -3.167198 & 1.775969 \\
\hline C & 1.530421 & -3.146140 & 2.918164 \\
\hline $\mathrm{N}$ & 0.167986 & -3.116333 & 2.775742 \\
\hline $\mathrm{N}$ & 2.343889 & -3.177406 & -1.282500 \\
\hline $\mathrm{H}$ & 3.626160 & -0.142146 & 3.054247 \\
\hline $\mathrm{H}$ & -0.513844 & -3.077465 & 3.523373 \\
\hline $\mathrm{H}$ & -1.048220 & 0.164068 & 1.715655 \\
\hline $\mathrm{H}$ & 5.330762 & -0.374475 & 1.193381 \\
\hline $\mathrm{H}$ & -2.034625 & -3.055741 & -1.109502 \\
\hline $\mathrm{H}$ & 1.992480 & -3.152315 & 3.896097 \\
\hline $\mathrm{H}$ & 2.287344 & -2.979114 & -2.273633 \\
\hline $\mathrm{H}$ & 3.194424 & -2.883028 & -0.816593 \\
\hline $\mathrm{H}$ & 0.402716 & -0.270723 & -2.509915 \\
\hline $\mathrm{H}$ & 2.121429 & -0.300800 & -2.446202 \\
\hline \multicolumn{4}{|c|}{28} \\
\hline \multicolumn{4}{|c|}{ A|C-Dimer-Twisted-pcm } \\
\hline $\mathrm{N}$ & 0.050077 & 0.045670 & 0.658950 \\
\hline $\mathrm{C}$ & -0.273751 & -0.198967 & 1.938577 \\
\hline $\mathrm{H}$ & 0.568719 & -0.315821 & 2.616331 \\
\hline $\mathrm{C}$ & -0.937620 & 0.203981 & -0.234934 \\
\hline $\mathrm{C}$ & -2.271629 & 0.122471 & 0.213535 \\
\hline $\mathrm{C}$ & -2.450222 & -0.133305 & 1.572810 \\
\hline $\mathrm{N}$ & -1.482132 & -0.310176 & 2.482564 \\
\hline $\mathrm{N}$ & -3.484007 & 0.298265 & -0.424419 \\
\hline $\mathrm{C}$ & -4.368853 & 0.144693 & 0.530774 \\
\hline $\mathrm{N}$ & -3.808024 & -0.118469 & 1.753118 \\
\hline $\mathrm{C}$ & -0.551810 & 2.954061 & 2.433235 \\
\hline $\mathrm{N}$ & 0.751242 & 3.024186 & 2.061811 \\
\hline $\mathrm{C}$ & 1.156754 & 3.211909 & 0.737230 \\
\hline $\mathrm{N}$ & 0.185984 & 3.346075 & -0.207170 \\
\hline C & -1.091898 & 3.271164 & 0.146015 \\
\hline C & -1.527832 & 3.078614 & 1.501424 \\
\hline
\end{tabular}




\begin{tabular}{|c|c|c|c|}
\hline b & 2.365669 & 3.251123 & 0.488183 \\
\hline & -2.013701 & 3.393463 & -0.828378 \\
\hline & -0.623577 & 0.410444 & -1.535501 \\
\hline & -5.441150 & 0.210982 & 0.407020 \\
\hline & -0.746958 & 2.795779 & 3.487691 \\
\hline & -2.577393 & 3.026216 & 1.763208 \\
\hline & -1.701876 & 3.440014 & -1.789623 \\
\hline & -2.983936 & 3.166304 & -0.657817 \\
\hline & -4.305082 & -0.267252 & 2.622946 \\
\hline & 1.489083 & 2.920253 & 2.748868 \\
\hline & 0.310493 & 0.750849 & -1.729758 \\
\hline & -1.354740 & 0.752769 & -2.146059 \\
\hline & & & \\
\hline \multicolumn{4}{|c|}{ A|G-Dimer-Twisted-pcm } \\
\hline & -0.047098 & 0.158635 & -0.197559 \\
\hline & -0.221176 & 0.270916 & 1.130069 \\
\hline & 0.691940 & 0.204459 & 2.093048 \\
\hline & 1.921073 & -0.001924 & 1.596761 \\
\hline & 2.258131 & -0.129870 & 0.248935 \\
\hline & 1.191375 & -0.045685 & -0.668594 \\
\hline & 3.108694 & -0.134274 & 2.265500 \\
\hline & 4.075819 & -0.342182 & 1.318405 \\
\hline & 3.612362 & -0.342769 & 0.092310 \\
\hline & 1.382502 & -0.132634 & -2.008793 \\
\hline & 0.033020 & -3.012993 & 1.327219 \\
\hline & 1.196066 & -3.207594 & 0.589809 \\
\hline & 1.109179 & -3.194478 & -0.838970 \\
\hline & -0.221967 & -2.998600 & -1.254179 \\
\hline & -1.307996 & -2.820615 & -0.432255 \\
\hline & -1.231692 & -2.830893 & 0.877498 \\
\hline & 2.290090 & -3.346460 & 1.418167 \\
\hline & 1.796359 & -3.241015 & 2.625133 \\
\hline & 0.436377 & -3.046733 & 2.628966 \\
\hline & 2.002026 & -3.313842 & -1.674300 \\
\hline & -2.500380 & -2.574350 & -1.040476 \\
\hline & 3.244767 & -0.108681 & 3.268654 \\
\hline & -1.247303 & 0.430918 & 1.451613 \\
\hline & -0.151159 & -2.908420 & 3.442202 \\
\hline & 5.111636 & -0.483861 & 1.595119 \\
\hline & 2.360099 & -3.297263 & 3.546092 \\
\hline & -0.351842 & -2.959132 & -2.261130 \\
\hline & -3.309347 & -2.654040 & -0.436829 \\
\hline & -2.646735 & -2.903980 & -1.986261 \\
\hline & 0.555915 & -0.278003 & -2.575570 \\
\hline $\mathrm{i}$ & 2.222416 & -0.597446 & -2.331751 \\
\hline 3 & & & \\
\hline \multicolumn{4}{|c|}{ A|T-Dimer-Twisted-pcm } \\
\hline $\mathrm{N}$ & 0.033991 & -0.116340 & 0.817740 \\
\hline C & 0.409475 & 0.078935 & 2.092366 \\
\hline N & 1.637215 & 0.200485 & 2.586638 \\
\hline $\mathrm{C}$ & 2.567396 & 0.111928 & 1.624349 \\
\hline C & 2.334711 & -0.083430 & 0.263090 \\
\hline $\mathrm{C}$ & 0.983643 & -0.200974 & -0.126887 \\
\hline $\mathrm{N}$ & 3.930182 & 0.188375 & 1.735839 \\
\hline c & 4.441672 & 0.040315 & 0.473200 \\
\hline N & 3.519485 & -0.126390 & -0.443995 \\
\hline & -0.404298 & 0.135569 & 2.811764 \\
\hline
\end{tabular}




$\begin{array}{lrrr}\mathrm{C} & -1.209808 & -3.084238 & 0.925922 \\ \mathrm{~N} & -0.644397 & -3.006751 & 2.173997 \\ \mathrm{C} & 0.712040 & -3.051195 & 2.396916 \\ \mathrm{C} & 1.609353 & -3.199683 & 1.398390 \\ \mathrm{C} & 1.099364 & -3.316906 & 0.036090 \\ \mathrm{~N} & -0.288758 & -3.267106 & -0.083962 \\ \mathrm{O} & -2.414310 & -3.015733 & 0.724716 \\ \mathrm{C} & 3.092107 & -3.250295 & 1.608016 \\ \mathrm{O} & 1.794570 & -3.447922 & -0.965397 \\ \mathrm{H} & 4.461072 & 0.324438 & 2.587640 \\ \mathrm{H} & -1.284592 & -2.868020 & 2.947178 \\ \mathrm{~N} & 0.618255 & -0.365976 & -1.416954 \\ \mathrm{H} & 5.507664 & 0.063994 & 0.292508 \\ \mathrm{H} & 1.009117 & -2.953920 & 3.435608 \\ \mathrm{H} & 3.597562 & -2.533303 & 0.952518 \\ \mathrm{H} & 3.343801 & -3.015793 & 2.645535 \\ \mathrm{H} & 3.489425 & -4.242330 & 1.367816 \\ \mathrm{H} & -0.669527 & -3.347062 & -1.022643 \\ \mathrm{H} & -0.343922 & -0.616560 & -1.606168 \\ \mathrm{H} & 1.312624 & -0.684204 & -2.080598\end{array}$

\section{References}

1 G. Barone, C. Fonseca Guerra and F. M. Bickelhaupt, ChemistryOpen, 2013, 2, 186-193.

2 X.-J. Lu and W. K. Olson, Nucleic Acids Res., 2003, 31, 5108-5121.

3 W. K. Olson, M. Bansal, S. K. Burley, R. E. Dickerson, M. Gerstein, S. C. Harvey, U. Heinemann, X.-J. Lu, S. Neidle, Z. Shakked, H. Sklenar, M. Suzuki, C.-S. Tung, E. Westhof, C. Wolberger and H. M. Berman, J. Mol. Biol., 2001, 313, 229-237.

4 C. Altona and M. Sundaralingam, J. Am. Chem. Soc., 1972, 94, 8205-8212.

5 D. Svozil, J. Kalina, M. Omelka and B. Schneider, Nucleic Acids Res., 2008, 36, 3690-3706. 